\title{
On Hamiltonian Paths and Cycles in Sufficiently Large Distance Graphs
}

\section{Christian Löwenstein吸 Dieter Rautenbach 非 Roman Soták $\left.\right|^{\mid \pi}$}

${ }^{1}$ Institut für Optimierung und Operations Research, Universität Ulm, Germany

${ }^{2}$ Faculty of Sciences, P.J. Šafárik University Košice, Slovakia

received $10^{\text {th }}$ Feb. 2011, revised 31 $31^{\text {st }}$ Aug. 2013, $4^{\text {th }}$ Dec. 2013, accepted $20^{\text {th }}$ Jan. 2014.

For a positive integer $n \in \mathbb{N}$ and a set $D \subseteq \mathbb{N}$, the distance graph $G_{n}^{D}$ has vertex set $\{0,1, \ldots, n-1\}$ and two vertices $i$ and $j$ of $G_{n}^{D}$ are adjacent exactly if $|j-i| \in D$. The condition $\operatorname{gcd}(D)=1$ is necessary for a distance graph $G_{n}^{D}$ being connected. Let $D=\left\{d_{1}, d_{2}\right\} \subseteq \mathbb{N}$ be such that $d_{1}>d_{2}$ and $\operatorname{gcd}\left(d_{1}, d_{2}\right)=1$. We prove the following results.

- If $n$ is sufficiently large in terms of $D$, then $G_{n}^{D}$ has a Hamiltonian path with endvertices 0 and $n-1$.

- If $d_{1} d_{2}$ is odd, $n$ is even and sufficiently large in terms of $D$, then $G_{n}^{D}$ has a Hamiltonian cycle.

- If $d_{1} d_{2}$ is even and $n$ is sufficiently large in terms of $D$, then $G_{n}^{D}$ has a Hamiltonian cycle.

Keywords: Distance graph; Toeplitz graph; circulant graph; Hamiltonian path; Hamiltonian cycle; traceability

\section{Introduction}

For a finite set of positive integers $D \subseteq \mathbb{N}$, the infinite distance graph $G^{D}$ has vertex set $V\left(G^{D}\right)=\mathbb{Z}$ and two vertices $u$ and $v$ of $G^{D}$ are adjacent exactly if $|u-v| \in D$. For a graph $G$ and a subset $U \subseteq V(G)$ of the vertex set, we denote by $G[U]$ the subgraph of $G$ induced by $U$. For $i, j \in \mathbb{Z}, i \leq j$, we denote by $[i, j]=\{k \in \mathbb{Z} \mid i \leq k \leq j\}$. For a positive integer $n \in \mathbb{N}$, the distance graph (also called Toeplitz graph in many papers) $G_{n}^{D}=G^{D}[[0, n-1]]$ is the subgraph of $G^{D}$ induced by the vertices in $[0, n-1]$.

Infinite distance graphs and especially their colourings were first studied by Eggleton, Erdős, and Skilton [10, 11]. Most of the research on distance graphs focused on their colourings [6, 8, 9, 14, 18, 19, 28]. Distance graphs generalize the very well-studied class of circulant graphs [2, 16, 17, 26]. In fact, circulant graphs coincide exactly with the regular distance graphs [23]. Circulant graphs have been proposed for numerous network applications and many of their properties such as connectedness and diameter [4, 2, 16, 17], cycle and path structure [1, 3, 5], and isomorphism testing and recognition [12, 22] have

\footnotetext{
‡Email: christian.loewenstein@uni-ulm.de

§Email: dieter.rautenbach@uni-ulm.de

IEmail: roman.sotak@upjs.sk
}

1365-8050 @ 2014 Discrete Mathematics and Theoretical Computer Science (DMTCS), Nancy, France 
been studied in great detail. Several fundamental results concerning circulant graphs were extended to the more general class of distance graphs in [7, 23, 24, 25]. The complexity of the connectedness problem for distance graphs was recently settled by Gómez et al. [13]. In [25, 27, 15] the existence of long paths and cycles in distance graphs is studied. The following main result from [21] confirmed a conjecture from Penso et al. [25]. [20] gives an overview on Hamiltonian cycles and paths in vertex-transitive graphs.

Theorem 1 (Löwenstein et al. [21]) For a finite set $D \subseteq \mathbb{N}$ with $|D| \geq 2$ and $\operatorname{gcd}(D)=1$, there are infinitely many $n \in \mathbb{N}$ such that $G_{n}^{D}$ has a Hamiltonian cycle and $G_{n+1}^{D}$ has a Hamiltonian path with endvertices 0 and $n$.

We conjecture that the conclusion of the last theorem holds

- for all $n$ that are sufficiently large in terms of $D$ if not all elements of $D$ are odd and

- for all even $n$ that are sufficiently large in terms of $D$ if all elements of $D$ are odd.

The purpose of the present paper is to confirm this conjecture in the case that $D$ contains just two elements. In Section 2 we introduce suitable terminology and collect some properties of distance graphs. In Section 3 we confirm our conjecture proving the existence of Hamiltonian paths. Finally, in Section 4 we provide similar results for Hamiltonian cycles.

\section{The structure of $G^{D}$}

Let $D=\left\{d_{1}, d_{2}\right\}$ for two positive integers $d_{1}$ and $d_{2}$ such that $\operatorname{gcd}\left(d_{1}, d_{2}\right)=1$ and $d_{1}>d_{2}$.

We define coordinates $(x, y) \in\left(\mathbb{Z} /\left(d_{1}+d_{2}\right) \mathbb{Z}\right) \times \mathbb{Z}$ for the vertices of the distance graph $G^{D}$ by

$$
(x, y):=y\left(d_{1}+d_{2}\right)+a_{x},
$$

where $a_{x}=x d_{1} \quad\left(\bmod d_{1}+d_{2}\right)$. Note that this bidimensional relabelling of the vertices of $G^{D}$ is a bijection. A vertex $(x, y)$ satisfying $0 \leq x d_{1} \quad\left(\bmod d_{1}+d_{2}\right)<d_{2}$ is called lower. A vertex $(x, y)$ satisfying $d_{2} \leq x d_{1}\left(\bmod d_{1}+d_{2}\right)<d_{1}$ is called middle. A vertex $(x, y)$ satisfying $d_{1} \leq x d_{1}$ $\left(\bmod d_{1}+d_{2}\right)<d_{1}+d_{2}$ is called upper.

For a lower vertex $(x, y)$, we have

$$
\begin{aligned}
& (x, y)+d_{1}=(x+1, y), \\
& (x, y)+d_{2}=(x-1, y), \\
& (x, y)-d_{1}=(x-1, y-1), \\
& (x, y)-d_{2}=(x+1, y-1),
\end{aligned}
$$

which implies that a lower vertex $(x, y)$ is adjacent to the vertices $(x+1, y),(x-1, y),(x+1, y-1)$, and $(x-1, y-1)$.

Similarly, for a middle vertex $(x, y)$, we have

$$
\begin{aligned}
& (x, y)+d_{1}=(x+1, y+1), \\
& (x, y)+d_{2}=(x-1, y), \\
& (x, y)-d_{1}=(x-1, y-1), \\
& (x, y)-d_{2}=(x+1, y),
\end{aligned}
$$


(a)

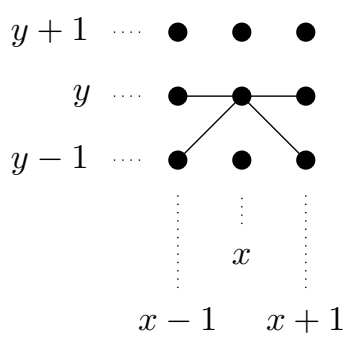

(b)

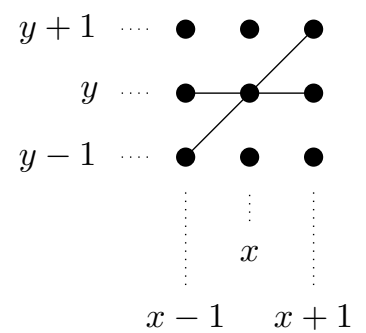

(c)

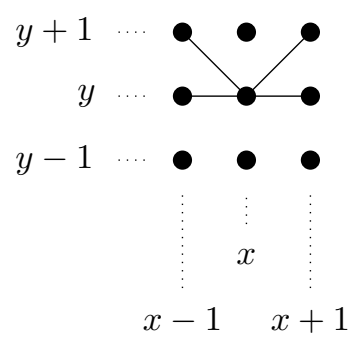

Fig. 1: Neighborhood of $(a)$ a lower, $(b)$ a middle, and $(c)$ an upper vertex.

which implies that a middle vertex $(x, y)$ is adjacent to the vertices $(x+1, y),(x-1, y),(x+1, y+1)$, and $(x-1, y-1)$.

Finally, for an upper vertex $(x, y)$, we have

$$
\begin{aligned}
& (x, y)+d_{1}=(x+1, y+1), \\
& (x, y)+d_{2}=(x-1, y+1), \\
& (x, y)-d_{1}=(x-1, y), \\
& (x, y)-d_{2}=(x+1, y),
\end{aligned}
$$

which implies that an upper vertex $(x, y)$ is adjacent to the vertices $(x+1, y),(x-1, y),(x+1, y+1)$, and $(x-1, y+1)$.

See Figure 1 for an illustration of these observations.

For $c \in \mathbb{Z} /\left(d_{1}+d_{2}\right) \mathbb{Z}$, all vertices $(x, y)$ of $G^{D}$ with $x=c$ form the column $c$. Similarly, for $r \in \mathbb{Z}$, all vertices $(x, y)$ satisfying $y=r$ form the row $r$. Note that the vertices in a column are either all lower, or all middle, or all upper. A column that consists of lower (middle, upper) vertices is called lower (middle, upper). See Figure 2 for an illustration.

Lemma 2 (i) For $c \in \mathbb{Z} /\left(d_{1}+d_{2}\right) \mathbb{Z}$, the column $c$ is lower if and only if the column $c+1$ is upper.

(ii) Column 0 is lower.

(iii) Column 1 is upper.

Proof: For $x \in \mathbb{Z} /\left(d_{1}+d_{2}\right) \mathbb{Z}$, we have $0 \leq x d_{1} \quad\left(\bmod d_{1}+d_{2}\right)<d_{2}$ if and only if $d_{1} \leq(x+1) d_{1}$ $\left(\bmod d_{1}+d_{2}\right)<d_{1}+d_{2}$, which proves (i). (ii) follows, because $0 \leq 0=0 d_{1} \quad\left(\bmod d_{1}+d_{2}\right)<d_{2}$. Finally, (i) and (ii) imply (iii).

The columns $x, x+1, \ldots, x+l-1$ form a block of length $l$, if column $x$ is lower, column $x+l$ is lower, and none of the columns $x+1, \ldots, x+l-1$ is lower. The block that contains column 0 is denoted by $B_{0}$. Let $l$ be the length of block $B_{i}$ and let column $x$ be the unique lower column that belongs to block 


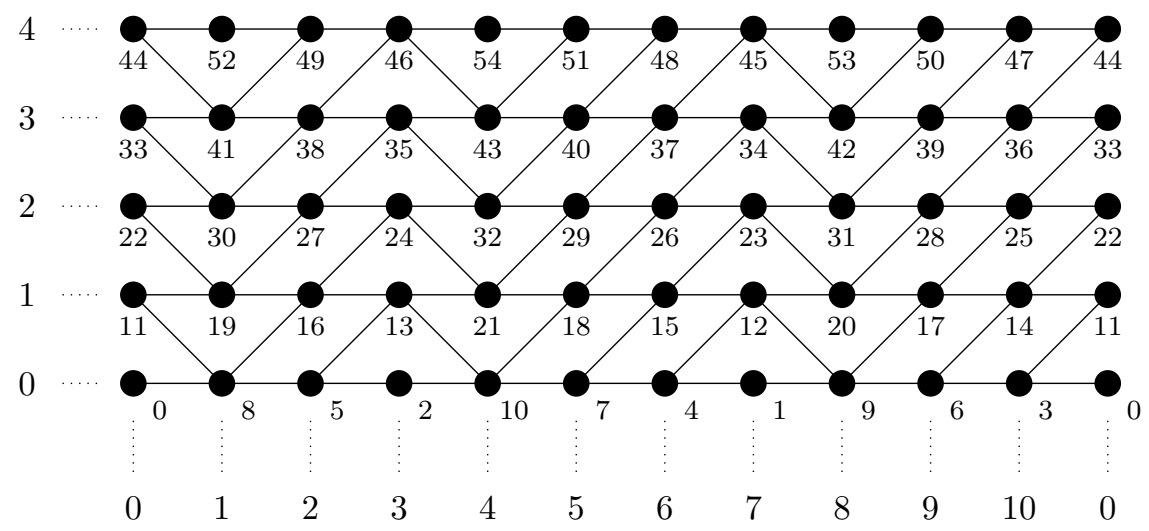

Fig. 2: The distance graph $G_{55}^{\{8,3\}}$. Note that the vertices of column 0 are drawn twice. In order to simplify the drawing, we adopt the convention that such a vertex is adjacent to the union of the neighbors of the two copies, i.e. vertex 22 is adjacent to the vertices $19,30,14$, and 25 .

$B_{i}$, then the block that contains column $x+l$ is denoted by $B_{i+1}$. Note that the indices of the blocks are elements of $\mathbb{Z} / d_{2} \mathbb{Z}$. For $i \in \mathbb{Z} / d_{2} \mathbb{Z}$, let $x_{i}$ denote the unique lower column in block $B_{i}$. Figure 3 shows the blocks of $G_{85}^{\{12,5\}}$.

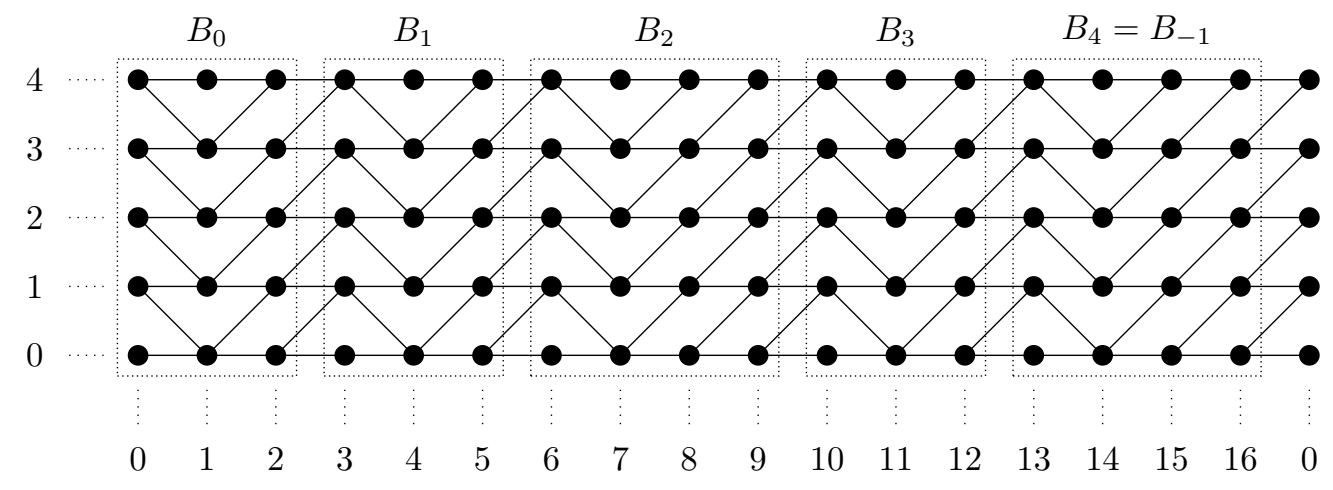

Fig. 3: Blocks of $G_{85}^{\{12,5\}}$. Note that 4 equals -1 in $\mathbb{Z} / 5 \mathbb{Z}$, that is, $B_{4}=B_{-1}$.

Lemma 3 (i) The length of a block is either $\left\lfloor\frac{d_{1}}{d_{2}}\right\rfloor+1$ or $\left\lceil\frac{d_{1}}{d_{2}}\right\rceil+1$.

(ii) The length of $B_{0}$ is $\left\lfloor\frac{d_{1}}{d_{2}}\right\rfloor+1$.

(iii) The length of $B_{-1}$ is $\left\lceil\frac{d_{1}}{d_{2}}\right\rceil+1$. 
(iv) The number of blocks is $d_{2}$.

Proof: Let $x, x+1, \ldots, x+l-1$ be the columns of a block $B$ of length $l$. By definition and Lemma 2 (i), $x$ is the unique lower column of block $B, x+1$ is the unique upper column of block $B$, and $x+l$ is a lower column. Hence, for all $y \in \mathbb{Z}$ and $x+1 \leq k \leq x+l-1$, we have $(k, y)-(k+1, y)=d_{2}$ and therefore $(x+1, y)-(x+l, y)=d_{2}(l-1)$. Since column $x+1$ is upper and column $x+l$ is lower, we have $d_{1}-d_{2}+1 \leq(x+1, y)-(x+l, y) \leq d_{1}+d_{2}-1$, which implies (i).

If $B=B_{0}$, then $x=0$ and $(x+1, y) \equiv d_{1} \quad\left(\bmod d_{1}+d_{2}\right)$ for all $y \in \mathbb{Z}$. Hence $(x+1, y)-(x+l, y) \leq$ $d_{1}$. Together with $(x+1, y)-(x+l, y)=d_{2}(l-1)$ this implies (ii).

If $B=B_{-1}$, then $x+l=0$ and $(x+l, y) \equiv 0 \quad\left(\bmod d_{1}+d_{2}\right)$ for all $y \in \mathbb{Z}$. Since column $x+1$ is upper, we have $(x+1, y)-(x+l, y) \geq d_{1}$. Together with $(x+1, y)-(x+l, y)=d_{2}(l-1)$ this implies (iii).

Since the function $f:\left\{0, \ldots, d_{1}+d_{2}-1\right\} \rightarrow\left\{0, \ldots, d_{1}+d_{2}-1\right\}$ with $f(x)=x d_{1} \quad\left(\bmod d_{1}+d_{2}\right)$ is bijective for $\operatorname{gcd}\left(d_{1}, d_{2}\right)=1$, there are exactly $d_{2}$ lower columns and therefore $d_{2}$ blocks, which proves (iv).

\section{Hamiltonian paths of $G_{n}^{D}$}

The main result of this section is the following.

Theorem 4 For every $D=\left\{d_{1}, d_{2}\right\} \subseteq \mathbb{N}$ with $d_{1}>d_{2}$ and $\operatorname{gcd}\left(d_{1}, d_{2}\right)=1$, there is some $n_{0} \in \mathbb{N}$ such that for all integers $n$ with $n \geq n_{0}$, the distance graph $G_{n}^{D}$ has a Hamiltonian path with endvertices 0 and $n-1$.

As before let $D=\left\{d_{1}, d_{2}\right\}$ for two positive integers $d_{1}$ and $d_{2}$ such that $\operatorname{gcd}\left(d_{1}, d_{2}\right)=1$ and $d_{1}>d_{2}$. For two lower vertices $(x, y)$ and $\left(x^{\prime}, y^{\prime}\right)$ with $x \neq x^{\prime}$ and $y<y^{\prime}$ in the distance graph $G^{D}$, a path in $G^{D}$ with endvertices $(x, y)$ and $\left(x^{\prime}, y^{\prime}\right)$ whose vertex set consists of all vertices in the rows $y, y+1, \ldots, y^{\prime}-1$ and the vertex $\left(x^{\prime}, y^{\prime}\right)$ is called an $(x, y)$ - $\left(x^{\prime}, y^{\prime}\right)$-climbing path of $G^{D}$. See Figure 5 for an illustration.

Before we proceed to the proof of Theorem 4 , we establish a series of lemmas concerning the existence of climbing paths.

Lemma 5 If $B_{i}$ is a block of even length in $G^{D}$, then $G^{D}$ has an $\left(x_{i}, y\right)-\left(x_{i+1}, y+2\right)$-climbing path for all $y$.

Proof: Let

$$
\begin{aligned}
P: \quad & \left(x_{i+1}-1, y\right),\left(x_{i+1}, y+1\right),\left(x_{i+1}-1, y+1\right),\left(x_{i+1}-2, y\right), \\
& \left(x_{i+1}-3, y\right),\left(x_{i+1}-2, y+1\right),\left(x_{i+1}-3, y+1\right),\left(x_{i+1}-4, y\right), \\
& \ldots,\left(x_{i}+3, y\right),\left(x_{i}+4, y+1\right),\left(x_{i}+3, y+1\right),\left(x_{i}+2, y\right) .
\end{aligned}
$$

The sequence

$$
\begin{aligned}
& \left(x_{i}, y\right),\left(x_{i}-1, y\right), \ldots,\left(x_{i+1}, y\right) \\
& P,\left(x_{i}+1, y\right) \\
& \left(x_{i}+2, y+1\right),\left(x_{i}+1, y+1\right), \ldots,\left(x_{i+1}+1, y+1\right), \\
& \left(x_{i+1}, y+2\right)
\end{aligned}
$$




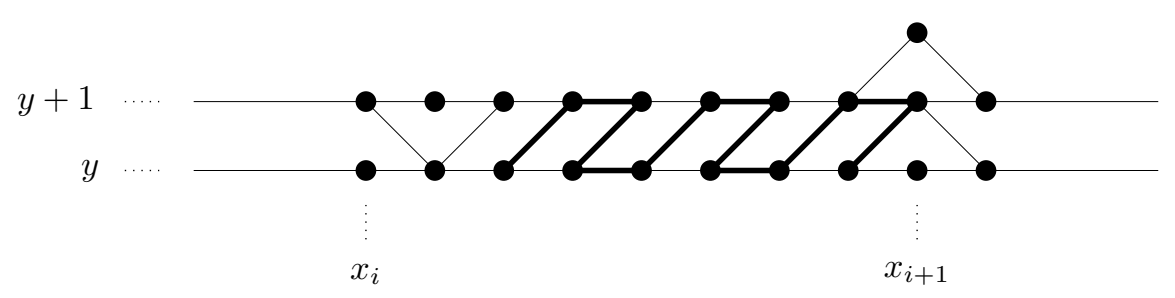

Fig. 4: $P$ for a block $B_{i}$ of length 8 .

defines an $\left(x_{i}, y\right)-\left(x_{i+1}, y+2\right)$-climbing path in $G^{D}$. See Figures 4 and 5 for an illustration.

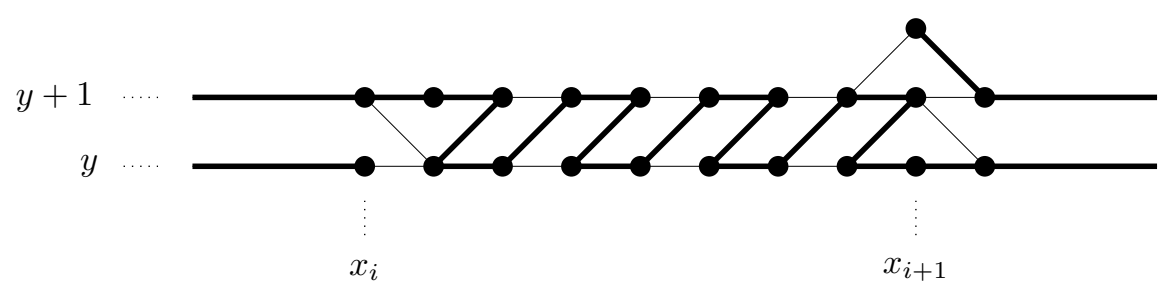

Fig. 5: An $\left(x_{i}, y\right)-\left(x_{i+1}, y+2\right)$-climbing path for block $B_{i}$ of length 8 .

Lemma 6 If $B_{i-1}$ is a block of even length in $G^{D}$, then $G^{D}$ has an $\left(x_{i}, y\right)-\left(x_{i-1}, y+2\right)$-climbing path for all $y$.

Proof: Let

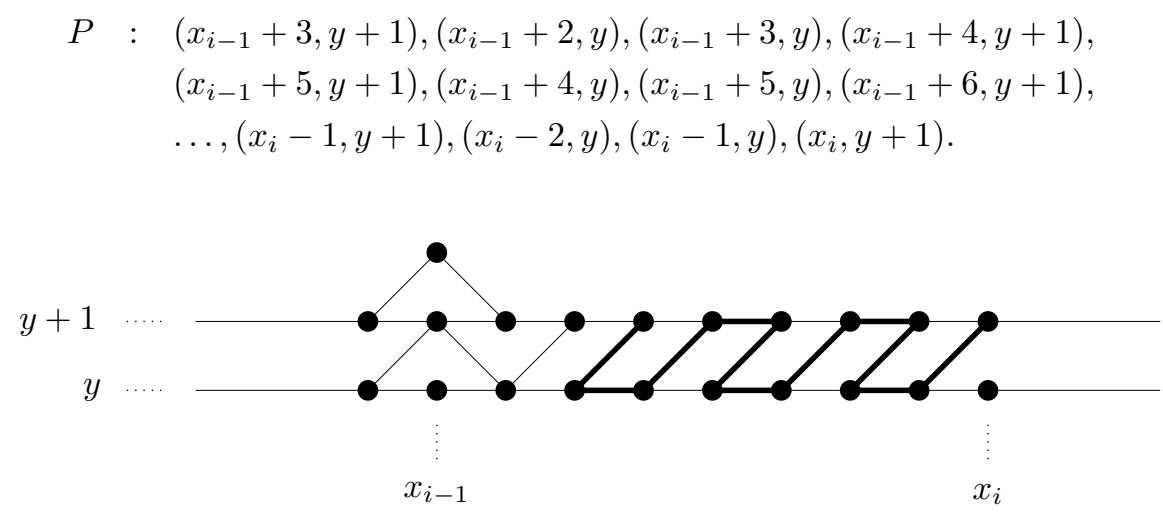

Fig. 6: $P$ for a block $B_{i-1}$ of length 8 . 
The sequence

$$
\begin{aligned}
& \left(x_{i}, y\right),\left(x_{i}+1, y\right), \ldots,\left(x_{i-1}+1, y\right), \\
& \left(x_{i-1}, y+1\right),\left(x_{i-1}+1, y+1\right),\left(x_{i-1}+2, y+1\right), P, \\
& \left(x_{i}+1, y+1\right),\left(x_{i}+2, y+1\right), \ldots,\left(x_{i-1}-1, y+1\right), \\
& \left(x_{i-1}, y+2\right)
\end{aligned}
$$

defines an $\left(x_{i}, y\right)-\left(x_{i-1}, y+2\right)$-climbing path of $G^{D}$. See Figures 6 and 7 for an illustration.

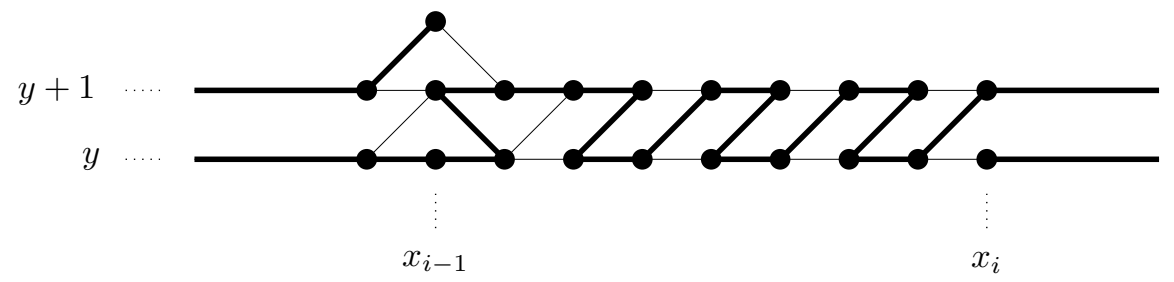

Fig. 7: An $\left(x_{i}, y\right)-\left(x_{i-1}, y+2\right)$-climbing path for block $B_{i-1}$ of length 8 .

Lemma 7 If $G^{D}$ has at least $j+2$ blocks for some $j \geq 1$ and for some $i \in \mathbb{Z} / d_{2} \mathbb{Z}$, the blocks $B_{i}, B_{i+1}, \ldots, B_{i+j}$ of $G^{D}$ are such that $B_{i}$ and $B_{i+j}$ are of odd length and $B_{i+1}, \ldots, B_{i+j-1}$ are of even length at least 4 , then $G^{D}$ has an $\left(x_{i}, y\right)-\left(x_{i+j+1}, y+3\right)$-climbing path for all $y$.

Proof: By Lemma 3 the blocks $B_{i}$ and $B_{i+j}$ are of length at least 3 .

Let

$$
\begin{aligned}
P_{i+j}: & \left(x_{i+j+1}-1, y\right),\left(x_{i+j+1}, y+1\right),\left(x_{i+j+1}-1, y+1\right),\left(x_{i+j+1}-2, y\right), \\
& \left(x_{i+j+1}-3, y\right),\left(x_{i+j+1}-2, y+1\right),\left(x_{i+j+1}-3, y+1\right),\left(x_{i+j+1}-4, y\right), \\
& \ldots,\left(x_{i+j}+2, y\right),\left(x_{i+j}+3, y+1\right),\left(x_{i+j}+2, y+1\right),\left(x_{i+j}+1, y\right) .
\end{aligned}
$$

For $1 \leq q \leq j-1$, let

$$
\begin{aligned}
P_{i+q}: \quad & \left(x_{i+q}+3, y+2\right),\left(x_{i+q}+2, y+1\right),\left(x_{i+q}+3, y+1\right),\left(x_{i+q}+4, y+2\right), \\
& \left(x_{i+q}+5, y+2\right),\left(x_{i+q}+4, y+1\right),\left(x_{i+q}+5, y+1\right),\left(x_{i+q}+6, y+2\right), \\
& \ldots,\left(x_{i+q+1}-3, y+2\right),\left(x_{i+q+1}-4, y+1\right),\left(x_{i+q+1}-3, y+1\right),\left(x_{i+q+1}-2, y+2\right)
\end{aligned}
$$

and let

$$
\begin{aligned}
P_{i+q}^{\prime}: & P_{i+q},\left(x_{i+q+1}-1, y+2\right),\left(x_{i+q+1}-2, y+1\right),\left(x_{i+q+1}-1, y+1\right),\left(x_{i+q+1}, y+1\right), \\
& \left(x_{i+q+1}+1, y+1\right),\left(x_{i+q+1}, y+2\right),\left(x_{i+q+1}+1, y+2\right),\left(x_{i+q+1}+2, y+2\right) .
\end{aligned}
$$

Note that $P_{i+q}$ is empty if $B_{i+q}$ is of length 4 . Furthermore, let

$$
\begin{aligned}
P_{i}: & \left(x_{i+1}-2, y+2\right),\left(x_{i+1}-3, y+1\right),\left(x_{i+1}-4, y+1\right),\left(x_{i+1}-3, y+2\right), \\
& \left(x_{i+1}-4, y+2\right),\left(x_{i+1}-5, y+1\right),\left(x_{i+1}-6, y+1\right),\left(x_{i+1}-5, y+2\right), \\
& \ldots,\left(x_{i}+3, y+2\right),\left(x_{i}+2, y+1\right),\left(x_{i}+1, y+1\right),\left(x_{i}+2, y+2\right) .
\end{aligned}
$$


Note that $P_{i}$ is empty if $B_{i}$ is of length 3 .

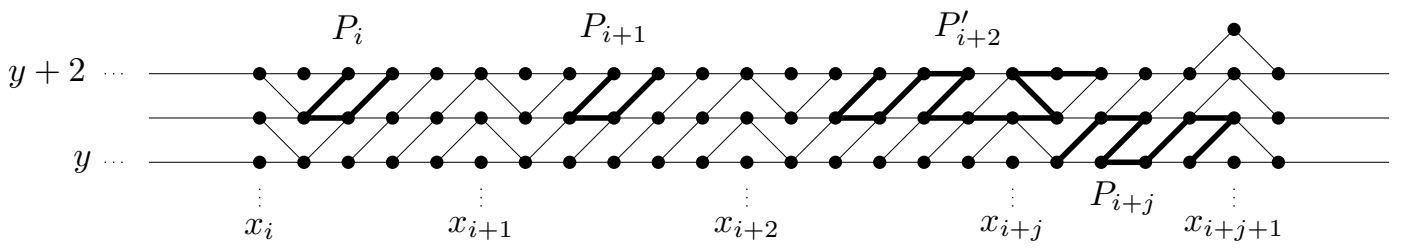

Fig. 8: $P_{i}, P_{i+1}, P_{i+2}^{\prime}$, and $P_{i+j}$ for $j=3$.

Now, the sequence

$$
\begin{aligned}
& \left(x_{i}, y\right),\left(x_{i}-1, y\right), \ldots,\left(x_{i+j+1}, y\right), P_{i+j}, \\
& \left(x_{i+j}, y\right),\left(x_{i+j}-1, y\right), \ldots,\left(x_{i+1}-1, y\right), \\
& \left(x_{i+1}, y+1\right),\left(x_{i+1}+1, y+1\right),\left(x_{i+1}, y+2\right),\left(x_{i+1}+1, y+2\right),\left(x_{i+1}+2, y+2\right), \\
& P_{i+1}^{\prime}, P_{i+2}^{\prime}, \ldots, P_{i+j-1}^{\prime}, \\
& \left(x_{i+j}+3, y+2\right),\left(x_{i+j}+4, y+2\right), \ldots,\left(x_{i+j+1}, y+2\right), \\
& \left(x_{i+j+1}+1, y+1\right),\left(x_{i+j+1}+2, y+1\right), \ldots,\left(x_{i}, y+1\right), \\
& \left(x_{i}+1, y\right),\left(x_{i}+2, y\right), \ldots,\left(x_{i+1}-2, y\right), \\
& \left(x_{i+1}-1, y+1\right),\left(x_{i+1}-2, y+1\right),\left(x_{i+1}-1, y+2\right), P_{i}, \\
& \left(x_{i}+1, y+2\right),\left(x_{i}, y+2\right), \ldots,\left(x_{i+j+1}+1, y+2\right), \\
& \left(x_{i+j+1}, y+3\right)
\end{aligned}
$$

defines an $\left(x_{i}, y\right)-\left(x_{i+j+1}, y+3\right)$-climbing path of $G^{D}$. See Figures 8 and 9 for an illustration.

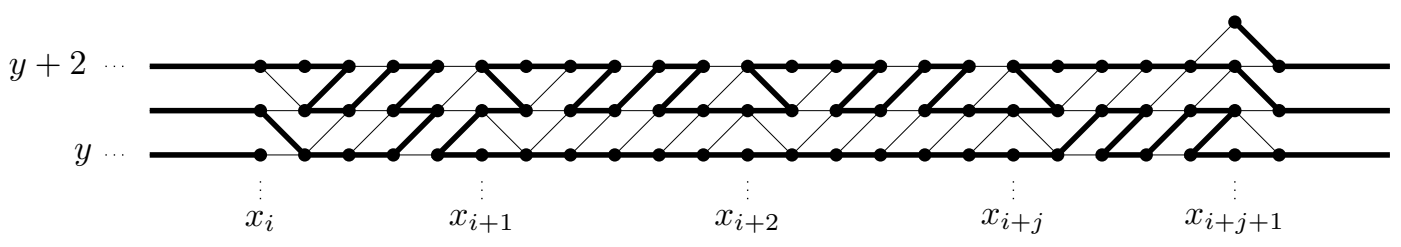

Fig. 9: An $\left(x_{i}, y\right)-\left(x_{i+j+1}, y+3\right)$-climbing path for $j=3$.

Lemma 8 If $G^{D}$ has at least $j+2$ blocks for some $j \geq 1$ and for some $i \in \mathbb{Z} / d_{2} \mathbb{Z}$, the blocks $B_{i}, B_{i+1}, \ldots, B_{i+j}$ of $G^{D}$ are such that $B_{i}$ and $B_{i+j}$ are of length 3 and $B_{i+1}, \ldots, B_{i+j-1}$ are of length 2 , then $G^{D}$ has an $\left(x_{i}, y\right)-\left(x_{i+j+1}, y+j+2\right)$-climbing path for all $y$.

Proof: Note that $x_{i+j+1}=x_{i}+2 j+4$. For $1 \leq q \leq j-1$, let

$$
P_{q}:\left(x_{i}+2 j+2, y+q\right),\left(x_{i}+2 j+3, y+q+1\right),\left(x_{i}+2 j+4, y+q+2\right),
$$




$$
\begin{aligned}
& \left(x_{i}+2 j+5, y+q+1\right),\left(x_{i}+2 j+6, y+q+1\right), \ldots,\left(x_{i}, y+q+1\right), \\
& \left(x_{i}+1, y+q\right),\left(x_{i}+2, y+q\right), \ldots,\left(x_{i}+2 j-2 q+2, y+q\right), \\
& \left(x_{i}+2 j-2 q+1, y+q+1\right),\left(x_{i}+2 j-2 q+2, y+q+1\right), \ldots,\left(x_{i}+2 j+1, y+q+1\right) .
\end{aligned}
$$

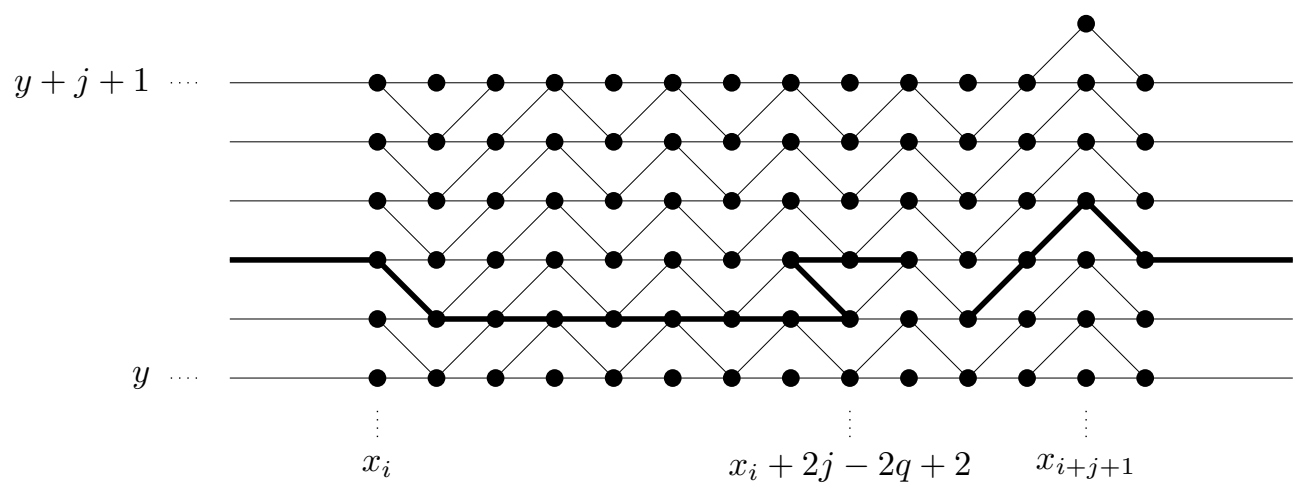

Fig. 10: $P_{q}$ for $j=4$ and $q=1$.

Now, the sequence

$$
\begin{aligned}
& \left(x_{i}, y\right),\left(x_{i}-1, y\right), \ldots,\left(x_{i}+2 j+4, y\right), \\
& \left(x_{i}+2 j+3, y\right),\left(x_{i}+2 j+4, y+1\right),\left(x_{i}+2 j+3, y+1\right),\left(x_{i}+2 j+4, y+2\right), \\
& \left(x_{i}+2 j+5, y+1\right),\left(x_{i}+2 j+6, y+1\right), \ldots,\left(x_{i}, y+1\right), \\
& \left(x_{i}+1, y\right),\left(x_{i}+2, y\right), \ldots,\left(x_{i}+2 j+2, y\right), \\
& \left(x_{i}+2 j+1, y+1\right) \\
& P_{1}, P_{2}, \ldots, P_{j-1} \\
& \left(x_{i}+2 j+2, y+j\right) \\
& \left(x_{i}+2 j+3, y+j+1\right)\left(x_{i}+2 j+2, y+j+1\right), \ldots,\left(x_{i}+3, y+j+1\right), \\
& \left(x_{i}+2, y+j\right),\left(x_{i}+1, y+j\right), \\
& \left(x_{i}+2, y+j+1\right)\left(x_{i}+1, y+j+1\right), \ldots,\left(x_{i}+2 j+5, y+j+1\right), \\
& \left(x_{i}+2 j+4, y+j+2\right)
\end{aligned}
$$

defines an $\left(x_{i}, y\right)-\left(x_{i+j+1}, y+j+2\right)$-climbing path of $G^{D}$. See Figures 10 and 11 for an illustration.

We are now in a position to prove the main result of this section. A path $P$ in $G^{D}$ with $V(P)=$ $[\min (V(P)), \max (V(P))]$ is called special, if the endvertices of $P$ are $\min (V(P))$ and $\max (V(P))$.

Proof of Theorem 4: If $d_{2}=1$, then the statement of the theorem is trivial. Hence we assume that $d_{2}>1$. The idea of the proof is to show the existence of two distinct positive integers $p_{1}$ and $p_{2}$ with 


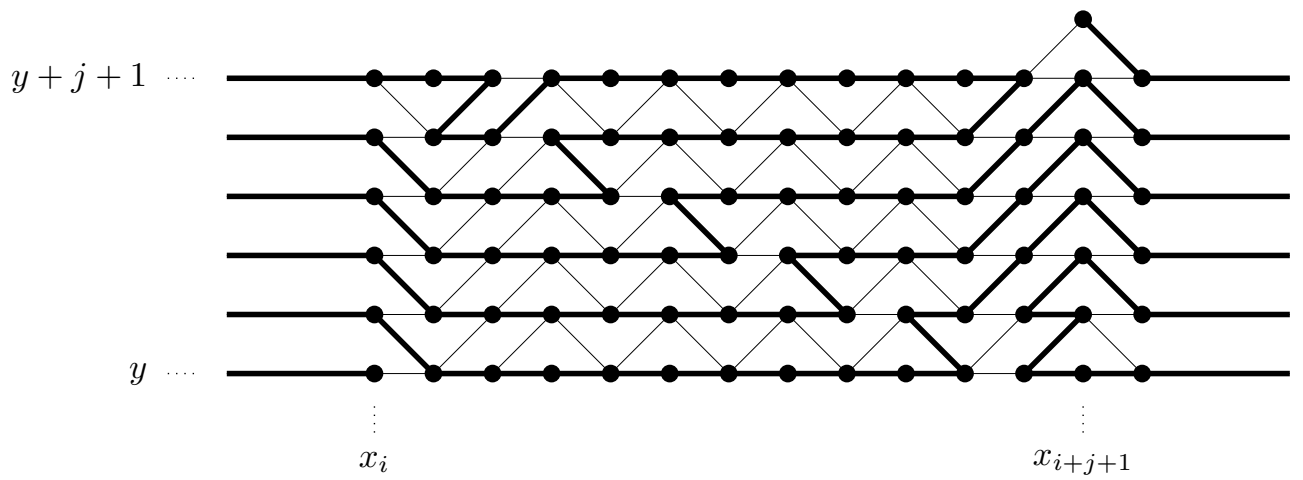

Fig. 11: An $\left(x_{i}, y\right)-\left(x_{i+j+1}, y+j+2\right)$-climbing path for $j=4$.

$\operatorname{gcd}\left(p_{1}, p_{2}\right)=1$ such that the distance graph $G^{D}$ has a special path of length $p_{1}$ and a special path of length $p_{2}$. Note that we can shift special paths. If $P: v_{0}, \ldots, v_{l}$ is a special path, then also $P+h$ : $v_{0}+h, \ldots, v_{l}+h$ is a special path. Furthermore, we can concatenate special paths. If $P: v_{0}, \ldots, v_{l}$ is a special path of length $l$ and $Q: v_{l}, \ldots, v_{l+h}$ is a special path of length $h$, then $P Q: v_{0}, \ldots, v_{l+h}$ is a special path of length $l+h$. Since $\operatorname{gcd}\left(p_{1}, p_{2}\right)=1$, it follows from the extended Euclidean algorithm that every sufficiently large integer $p$ is a positive integral linear combination of $p_{1}$ and $p_{2}$. In fact, if $p>\left(2 p_{2}-1\right) p_{1}$ and $p=a_{1} p_{1}+a_{2} p_{2}$ for integers $a_{1}$ and $a_{2}$ such that $a_{1} \leq 0$, then $a_{1}+s p_{2}>0$ and $a_{2}-s p_{1}>0$ for $s=\left\lceil\frac{-a_{1}}{p_{2}}\right\rceil+1$ and thus $p=\left(a_{1}+s p_{2}\right) p_{1}+\left(a_{2}-s p_{1}\right) p_{2}$ is a positive integral linear combination of $p_{1}$ and $p_{2}$. Therefore, the desired result follows by shifting and concatenating copies of the special paths of lengths $p_{1}$ and $p_{2}$, which we construct now.

It has been observed in [25, 21] that $G_{d_{1}+d_{2}+1}^{D}$ has a Hamiltonian path with endvertices 0 and $d_{1}+d_{2}$. Hence, for $p_{1}$, we choose $d_{1}+d_{2}$.

For $p_{2}$, we show that there is a positive integer $p_{2}$ with $p_{2} \equiv-1 \quad\left(\bmod d_{1}+d_{2}\right) \equiv-1 \quad\left(\bmod p_{1}\right)$, such that $G^{D}$ has a special path of length $p_{2}$, thus $\operatorname{gcd}\left(p_{1}, p_{2}\right)=1$.

Let $x^{\prime}$ be such that $x^{\prime} d_{1} \equiv-1 \quad\left(\bmod d_{1}+d_{2}\right)$. By definition and Lemma2 (i), column $x^{\prime}$ is upper and column $x^{\prime}-1$ is lower. In order to get a special path with endvertices $(0,0)$ and $\left(x^{\prime}, y^{\prime}\right)$ for some $y^{\prime}$, we concatenate climbing paths to form a $(0,0)-\left(x^{\prime}-1, y^{\prime}\right)$-climbing path and append the path $\left(x^{\prime}-\right.$ $\left.2, y^{\prime}\right),\left(x^{\prime}-3, y^{\prime}\right), \ldots,\left(x^{\prime}, y^{\prime}\right)$.

Let $k$ be such that the block $B_{k}$ contains column $x^{\prime}$, that is, $x_{k}=x^{\prime}-1$. Since column $x^{\prime}$ is upper, column $x^{\prime}-2$ belongs to block $B_{k-1}$.

Since $\operatorname{gcd}\left(d_{1}, d_{2}\right)=1$, at least one of $d_{1}$ and $d_{2}$ is odd.

Case 1 One of $d_{1}$ and $d_{2}$ is even and $G^{D}$ has at most 2 blocks of odd length.

Since $d_{1}+d_{2}$ is odd, the number of blocks of odd length is odd, that is, it equals 1 .

We first assume that all blocks $B_{0}, B_{1}, \ldots, B_{k-1}$ are of even length. By Lemma 5 , there exists an $\left(x_{i}, 2 i\right)-\left(x_{i+1}, 2 i+2\right)$-climbing path $P_{i}$ for $0 \leq i \leq k-1$. Since $x^{\prime}-1=x_{k}$, the concatenation of the paths $P_{0}, P_{1}, \ldots, P_{k-1}$ forms a $(0,0)-\left(x^{\prime}-1, y^{\prime}\right)$-climbing path for $y^{\prime}=2 k$. 
Next, we assume that all blocks $B_{k}, B_{k+1}, \ldots, B_{-1}$ are of even length. Then, by Lemma 6 , there exists an $\left(x_{i+1}, 2\left(d_{1}+d_{2}-i\right)-2\right)-\left(x_{i}, 2\left(d_{1}+d_{2}-i\right)\right)$-climbing path $P_{i}$ for $k \leq i \leq d_{1}+d_{2}-1$. Since $x^{\prime}-1=x_{k}$, the concatenation of the paths $P_{d_{1}+d_{2}-1}, P_{d_{1}+d_{2}-2}, \ldots, P_{k}$ forms a $(0,0)-\left(x^{\prime}-1, y^{\prime}\right)-$ climbing path for $y^{\prime}=2\left(d_{1}+d_{2}-k\right)$. This concludes the first case.

Case 2 One of $d_{1}$ and $d_{2}$ is even and $G^{D}$ has at least 3 blocks of odd length.

Since $d_{1}+d_{2}$ is odd, the number of blocks of odd length is odd. This implies that one of the two sequences $B_{0}, B_{1}, \ldots, B_{k-1}$ and $B_{0}, B_{1}, \ldots, B_{-1}, B_{0}, B_{1}, \ldots, B_{k-1}$ has an even number of blocks with odd length. We call this sequence $\mathcal{S}$. We can partition $\mathcal{S}$ into subsequences $S_{1}, S_{2}, \ldots, S_{t}$, where each subsequence is either a block of even length or a sequence $B_{i}, B_{i+1}, \ldots, B_{i+j}$ of blocks with $i \in \mathbb{Z} / d_{2} \mathbb{Z}$ and $j \geq 1$, such that block $B_{i}$ has odd length, block $B_{i+j}$ has odd length, and blocks $B_{i+1}, \ldots, B_{i+j-1}$ have even length. For a subsequence $S_{q}, 1 \leq q \leq t$, that consists of one block $B_{i}$ with $i \in \mathbb{Z} / d_{2} \mathbb{Z}$, Lemma 5 implies that there exists an $\left(x_{i}, y\right)-\left(x_{i+1}, y+2\right)$-climbing path $P_{q, y}$ for every $y$. If $\frac{d_{1}}{d_{2}}<2$, then Lemma 3 implies that the lengths of the blocks are 2 and 3 . For a subsequence $S_{q}, 1 \leq q \leq t$, that consists of at least two blocks $B_{i}, B_{i+1}, \ldots, B_{i+j}$ with $i \in \mathbb{Z} / d_{2} \mathbb{Z}$ and $j \geq 1$, Lemma 8 implies that there exists an $\left(x_{i}, y\right)-\left(x_{i+j+1}, y+j+2\right)$-climbing path $P_{q, y}$ for every $y$. If $\frac{d_{1}}{d_{2}} \geq 2$, then Lemma 3 implies that the lengths of the blocks are at least 3 . For a subsequence $S_{q}, 1 \leq q \leq t$, that consists of at least two blocks $B_{i}, B_{i+1}, \ldots, B_{i+j}$ with $i \in \mathbb{Z} / d_{2} \mathbb{Z}$ and $j \geq 1$, Lemma 7 implies that there exists an $\left(x_{i}, y\right)-\left(x_{i+j+1}, y+3\right)$-climbing path $P_{q, y}$ for every $y$.

The concatenation of the paths $P_{1, y_{1}}, P_{2, y_{2}}, \ldots, P_{t, y_{t}}$ forms a $(0,0)-\left(x^{\prime}-1, y^{\prime}\right)$-climbing path for $y_{1}=0$, suitable $y_{q}$ 's, where $2 \leq q \leq t$, and $y^{\prime}=y_{t}$.

This concludes the second case.

If both $d_{1}$ and $d_{2}$ are odd, then $d_{1}+d_{2}$ is even, which implies that the number of blocks of odd length is even and exactly those vertices are even integers that are in a column with an even index. This implies that $x^{\prime}$ is odd and $x_{k}=x^{\prime}-1$ is even. Since column 0 and column $x^{\prime}-1$ are lower, the sequence $B_{0}, B_{1}, \ldots, B_{k-1}$ has an even number of blocks with odd length.

Case 3 Both $d_{1}$ and $d_{2}$ are odd and $G^{D}$ has at most 2 blocks of odd length.

Since $d_{2} \geq 2, G$ has exactly 2 blocks of odd length. This implies that one of the two sequences $B_{0}, B_{1}, \ldots, B_{k-1}$ and $B_{k}, B_{k+1}, \ldots, B_{-1}$ has only blocks of even length. Now we are in the same situation as in Case 1 . Arguing as in Case 1 completes this case.

Case 4 Both $d_{1}$ and $d_{2}$ are odd and $G^{D}$ has at least 4 blocks of odd length.

Since the sequence $B_{0}, B_{1}, \ldots, B_{k-1}$ has an even number of blocks of odd length, we are in the same situation as in Case 2 Arguing as in Case 2 completes this case, which concludes the proof of the theorem.

\section{Hamiltonian cycles of $G_{n}^{D}$}

The main results of this section are the following.

Theorem 9 For every $D=\left\{d_{1}, d_{2}\right\} \subseteq \mathbb{N}$ with $d_{1}>d_{2}, d_{1} d_{2}$ odd, and $\operatorname{gcd}\left(d_{1}, d_{2}\right)=1$, there is some $n_{0} \in \mathbb{N}$ such that for all even integers $n$ with $n \geq n_{0}$, the distance graph $G_{n}^{D}$ has a Hamiltonian cycle. 
Theorem 10 For every $D=\left\{d_{1}, d_{2}\right\} \subseteq \mathbb{N}$ with $d_{1}>d_{2}, d_{1} d_{2}$ even, and $\operatorname{gcd}\left(d_{1}, d_{2}\right)=1$, there is some $n_{0} \in \mathbb{N}$ such that for all integers $n$ with $n \geq n_{0}$, the distance graph $G_{n}^{D}$ has a Hamiltonian cycle.

Note that the distance graphs considered in Theorem 9 are necessarily bipartite. Therefore, they can only have a Hamiltonian cycle if their order is even.

As in Section 3 we establish several lemmas before proceeding to the proofs of Theorems 9 and 10

For two lower vertices $(x, y)$ and $\left(x^{\prime}, y^{\prime}\right)$ with $x \neq x^{\prime}, 0 \notin\left\{x^{\prime}+1, x^{\prime}+2, \ldots, x\right\}$, and $y<y^{\prime}$ in the distance graph $G^{D}$, a set of vertex disjoint paths $R_{y}, R_{y+1}, \ldots, R_{y^{\prime}-1}$ in $G^{D}$ is called an $(x, y)-\left(x^{\prime}, y^{\prime}\right)$ path-collection of $G^{D}$, if it satisfies the following conditions:

- for $y \leq i<y^{\prime}, P_{i}$ has the endvertices $(0, i)$ and $(-1, i+1)$,

- for $y \leq i<y^{\prime}$, the path $(0, i),(1, i), \ldots,\left(x^{\prime}, i\right)$ is a subpath of $P_{i}$,

- for $y \leq i<y^{\prime}$, the path $(x, i+1),(x+1, i+1), \ldots,(-1, i+1)$ is a subpath of $P_{i}$,

- the union of the vertex sets of the paths consists of all vertices in the rows $y+1, y+2, \ldots, y^{\prime}-1$, the vertices $\{(0, y),(1, y), \ldots,(x-1, y)\}$, and the vertices $\left\{\left(x^{\prime}, y^{\prime}\right),\left(x^{\prime}+1, y^{\prime}\right), \ldots,\left(-1, y^{\prime}\right)\right\}$, and

- no edge of the form $\left\{(-1, z),\left(0, z^{\prime}\right)\right\}$ for some $z, z^{\prime} \in \mathbb{Z}$ is in the union of the edge sets of the paths.

See Figures 13, 15 and 16 for an illustration. Note, that $(x, y)$ does not belong to any path of an $(x, y)$ $\left(x^{\prime}, y^{\prime}\right)$-path-collection.

Lemma 11 If for some $i \neq-1, B_{i}$ is a block of even length in $G^{D}$, then $G^{D}$ has an $\left(x_{i+1}, y\right)-\left(x_{i}, y+1\right)$ path collection for all $y$.

Proof: Let

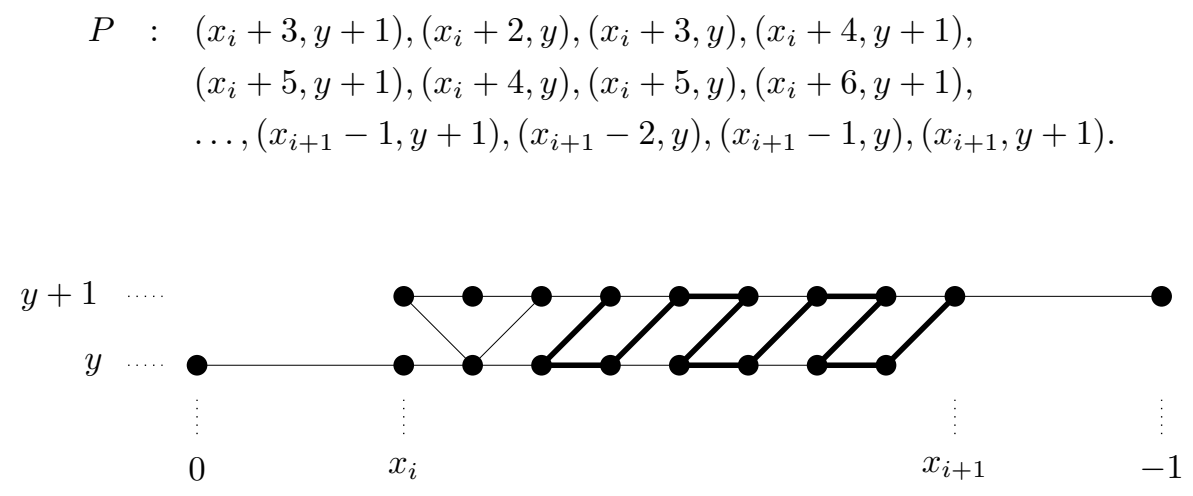

Fig. 12: $P$ for a block $B_{i}$ of length 8 . 
The sequence

$$
\begin{aligned}
& (0, y),(1, y), \ldots,\left(x_{i}+1, y\right) \\
& \left(x_{i}, y+1\right),\left(x_{i}+1, y+1\right),\left(x_{i}+2, y+1\right), P \\
& \left(x_{i+1}+1, y+1\right),\left(x_{i+1}+2, y+1\right), \ldots,(-1, y+1)
\end{aligned}
$$

defines an $\left(x_{i+1}, y\right)-\left(x_{i}, y+1\right)$-path-collection in $G^{D}$. See Figures 12 and 13 for an illustration.

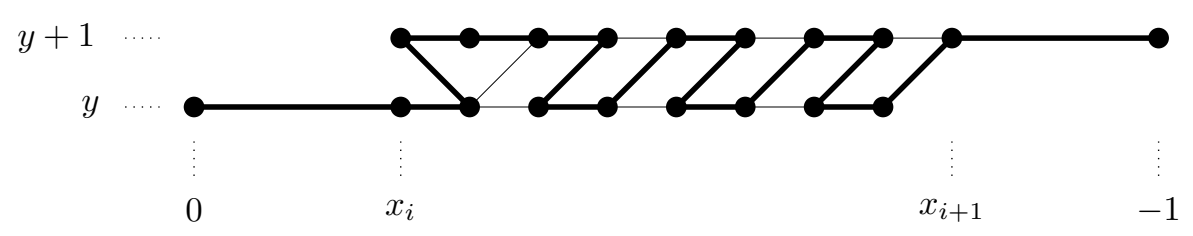

Fig. 13: An $\left(x_{i+1}, y\right)-\left(x_{i}, y+1\right)$-path-collection for a block $B_{i}$ of length 8 .

Lemma 12 If for some $i \in \mathbb{Z} / d_{2} \mathbb{Z}$ and for some $j \geq 1$, the blocks $B_{i}, B_{i+1}, \ldots, B_{i+j}$ of $G^{D}$ are such that $-1 \notin\{i, i+1, \ldots, i+j\}, B_{i}$ and $B_{i+j}$ are of odd length and $B_{i+1}, \ldots, B_{i+j-1}$ are of even length at least 4 , then $G^{D}$ has an $\left(x_{i+j+1}, y\right)-\left(x_{i}, y+2\right)$-path-collection for all $y$.

Proof: By Lemma 3 the blocks $B_{i}$ and $B_{i+j}$ are of length at least 3 .

Let

$$
\begin{aligned}
P_{i}: & \left(x_{i}+1, y\right),\left(x_{i}+2, y+1\right),\left(x_{i}+3, y+1\right),\left(x_{i}+2, y\right), \\
& \left(x_{i}+3, y\right),\left(x_{i}+4, y+1\right),\left(x_{i}+5, y+1\right),\left(x_{i}+4, y\right), \\
& \ldots,\left(x_{i+1}-2, y\right),\left(x_{i+1}-1, y+1\right),\left(x_{i+1}, y+1\right),\left(x_{i+1}-1, y\right) .
\end{aligned}
$$

For $1 \leq q \leq j-1$, let

$$
\begin{aligned}
P_{i+q}: & \left(x_{i+q}, y\right),\left(x_{i+q}+1, y\right) \\
& \left(x_{i+q}+2, y\right),\left(x_{i+q}+3, y+1\right),\left(x_{i+q}+4, y+1\right),\left(x_{i+q}+3, y\right) \\
& \left(x_{i+q}+4, y\right),\left(x_{i+q}+5, y+1\right),\left(x_{i+q}+6, y+1\right),\left(x_{i+q}+5, y\right) \\
& \ldots,\left(x_{i+q+1}-2, y\right),\left(x_{i+q+1}-1, y+1\right),\left(x_{i+q+1}, y+1\right),\left(x_{i+q+1}-1, y\right) .
\end{aligned}
$$

Furthermore, let

$$
\begin{aligned}
P_{i+j}: \quad & \left(x_{i+j}+2, y\right),\left(x_{i+j}+3, y+1\right),\left(x_{i+j}+4, y+1\right),\left(x_{i+j}+3, y\right), \\
& \left(x_{i+j}+4, y\right),\left(x_{i+j}+5, y+1\right),\left(x_{i+j}+6, y+1\right),\left(x_{i+j}+5, y\right), \\
& \ldots,\left(x_{i+j+1}-3, y\right),\left(x_{i+j+1}-2, y+1\right),\left(x_{i+j+1}-1, y+1\right),\left(x_{i+j+1}-2, y\right) .
\end{aligned}
$$

For $1 \leq q \leq j$, let

$$
\begin{aligned}
Q_{i+q}: & \left(x_{i+q-1}+4, y+2\right),\left(x_{i+q-1}+5, y+2\right), \ldots,\left(x_{i+q}+2, y+2\right), \\
& \left(x_{i+q}+1, y+1\right),\left(x_{i+q}+2, y+1\right),\left(x_{i+q}+3, y+2\right) .
\end{aligned}
$$




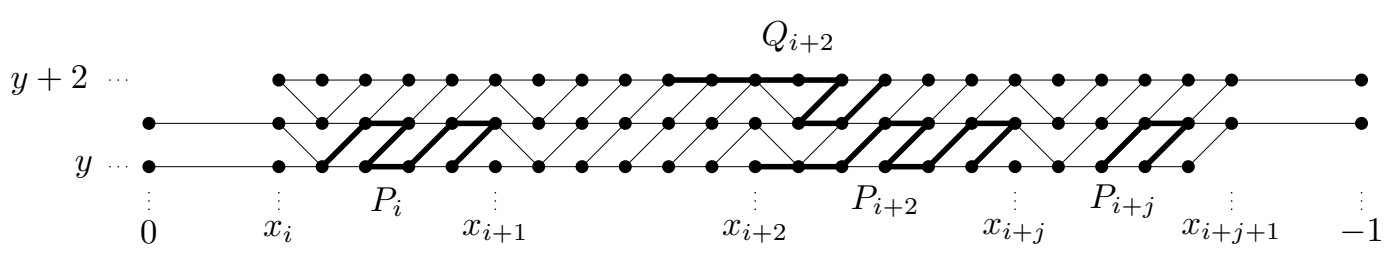

Fig. 14: $P_{i}, P_{i+2}, P_{i+j}$, and $Q_{i+2}$ for $j=3$.

Now, $R_{y}$ and $R_{y+1}$, where

$$
\begin{aligned}
R_{y}: & (0, y),(1, y), \ldots,\left(x_{i}, y\right), \\
& P_{i}, \\
& P_{i+1}, P_{i+2}, \ldots, P_{i+j-1}, \\
& \left(x_{i+j}, y\right),\left(x_{i+j}+1, y\right), P_{i+j},\left(x_{i+j+1}-1, y\right), \\
& \left(x_{i+j+1}, y+1\right),\left(x_{i+j+1}+1, y+1\right), \ldots,(-1, y+1)
\end{aligned}
$$

and

$$
\begin{aligned}
R_{y+1}: & (0, y+1),(1, y+1), \ldots,\left(x_{i}+1, y+1\right), \\
& \left(x_{i}, y+2\right),\left(x_{i}+1, y+2\right),\left(x_{i}+2, y+2\right),\left(x_{i}+3, y+2\right), \\
& Q_{i+1}, Q_{i+2}, \ldots, Q_{i+j}, \\
& \left(x_{i+j+1}-1, y+2\right),\left(x_{i+j+1}, y+2\right), \ldots,(-1, y+2)
\end{aligned}
$$

define an $\left(x_{i+j+1}, y\right)-\left(x_{i}, y+2\right)$-path-collection of $G^{D}$. See Figures 14 and 15 for an illustration.

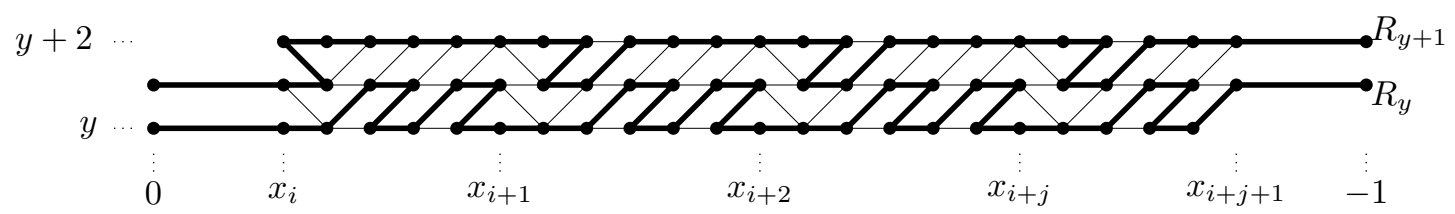

Fig. 15: An $\left(x_{i+j+1}, y\right)-\left(x_{i}, y+2\right)$-path-collection for $j=3$.

Lemma 13 If for some $i \in \mathbb{Z} / d_{2} \mathbb{Z}$ and for some $j \geq 1$, the blocks $B_{i}, B_{i+1}, \ldots, B_{i+j}$ of $G^{D}$ are such that $-1 \notin\{i, i+1, \ldots, i+j\}, B_{i}$ and $B_{i+j}$ are of length 3 and $B_{i+1}, \ldots, B_{i+j-1}$ are of length 2 , then $G^{D}$ has an $\left(x_{i+j+1}, y\right)-\left(x_{i}, y+j+1\right)$-path-collection for all $y$.

Proof: Note that $x_{i+j+1}=x_{i}+2 j+4$. For $0 \leq q \leq j-1$, let

$$
R_{i+q}:(0, y+q),(1, y+q), \ldots,\left(x_{i}+1, y+q\right),
$$




$$
\begin{aligned}
& \left(x_{i}+2, y+q+1\right),\left(x_{i}+3, y+q+1\right), \ldots,\left(x_{i}+2 q+3, y+q+1\right), \\
& \left(x_{i}+2 q+2, y+q\right),\left(x_{i}+2 q+3, y+q\right), \ldots,\left(x_{i}+2 j+3, y+q\right), \\
& \left(x_{i}+2 j+4, y+q+1\right),\left(x_{i}+2 j+5, y+q+1\right), \ldots,(-1, y+q+1) .
\end{aligned}
$$

and let

$$
\begin{aligned}
R_{i+j}: \quad & (0, y+j),(1, y+j), \ldots,\left(x_{i}+1, y+j\right), \\
& \left(x_{i}, y+j+1\right),\left(x_{i}+1, y+j+1\right), \ldots,\left(x_{i}+2 j+3, y+j+1\right), \\
& \left(x_{i}+2 j+2, y+j\right),\left(x_{i}+2 j+3, y+j\right), \\
& \left(x_{i}+2 j+4, y+j+1\right),\left(x_{i}+2 j+5, y+j+1\right), \ldots,(-1, y+j+1) .
\end{aligned}
$$

Now, $R_{i}, R_{i+1}, \ldots, R_{i+q}$ define an $\left(x_{i+j+1}, y\right)-\left(x_{i}, y+j+1\right)$-path-collection of $G^{D}$. See Figure 16 for an illustration.

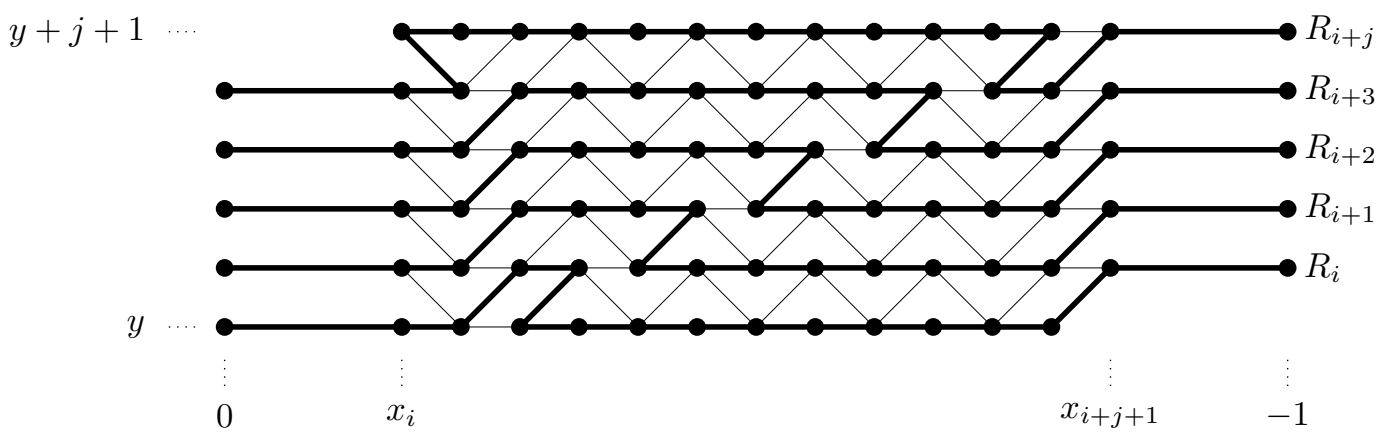

Fig. 16: An $\left(x_{i+j+1}, y\right)-\left(x_{i}, y+j+1\right)$-path-collection for $j=4$.

Lemma 14 If for some $i \in \mathbb{Z} / d_{2} \mathbb{Z}$ and for some $j \geq 0$, the sequence $\mathcal{S}=B_{i}, B_{i+1}, \ldots, B_{i+j}$ of blocks of $G^{D}$ is such that $-1 \notin\{i, i+1, \ldots, i+j\}$ and the number of blocks of odd length among $B_{i}, B_{i+1}, \ldots, B_{i+j}$ is even, then $G^{D}$ has an $\left(x_{i+j+1}, y\right)-\left(x_{i}, y+\Delta y\right)$-path-collection for some $\Delta y$ and for all $y$.

Proof: By definition, the union of suitable path-collections is a path-collection: If for some $x, x^{\prime}, x^{\prime \prime}, y$, $y^{\prime}, y^{\prime \prime}, G^{D}$ has an $(x, y)-\left(x^{\prime}, y^{\prime}\right)$-path-collection and an $\left(x^{\prime}, y^{\prime}\right)$ - $\left(x^{\prime \prime}, y^{\prime \prime}\right)$-path-collection, then $G^{D}$ has an $(x, y)$ - $\left(x^{\prime \prime}, y^{\prime \prime}\right)$-path-collection. We can partition $\mathcal{S}$ into subsequences, where each subsequence is either a block of even length or a sequence $B_{k}, B_{k+1}, \ldots, B_{k+l}$ of blocks with $k \in \mathbb{Z} / d_{2} \mathbb{Z}$ and $l \geq 1$, such that blocks $B_{k}$ and $B_{k+l}$ have odd length and blocks $B_{k+1}, \ldots, B_{k+l-1}$ have even length. For a subsequence that consists of one even block $B_{k}$ with $k \in \mathbb{Z} / d_{2} \mathbb{Z}$, Lemma 11 implies that there exists a $\left(x_{k+1}, y\right)$ $\left(x_{k}, y+1\right)$ path collection for every $y$. If $\frac{d_{1}}{d_{2}}<2$, then Lemma 3 implies that the lengths of the blocks are 2 and 3 . For a subsequence that consists of at least two blocks $B_{k}, B_{k+1}, \ldots, B_{k+l}$ with $k \in \mathbb{Z} / d_{2} \mathbb{Z}$ and $l \geq 1$, Lemma 13 implies that there exists an $\left(x_{k+l+1}, y\right)-\left(x_{k}, y+l+1\right)$-path-collection for every $y$. If $\frac{d_{1}}{d_{2}} \geq 2$, then Lemma 3 implies that the lengths of the blocks are at least 3 . For a subsequence that consists 
of at least two blocks $B_{k}, B_{k+1}, \ldots, B_{k+l}$ with $k \in \mathbb{Z} / d_{2} \mathbb{Z}$ and $l \geq 1$, Lemma 12 implies that there exists an $\left(x_{k+l+1}, y\right)-\left(x_{k}, y+2\right)$-path-collection for every $y$. Hence, a suitable union of path-collections forms an $\left(x_{i+j+1}, y\right)-\left(x_{i}, y+\Delta y\right)$-path-collection for a suitable $\Delta y$ and all $y$.

Lemma 15 If for some $-i \in \mathbb{Z} / d_{2} \mathbb{Z}$, the blocks $B_{-i}, B_{-i+1}, \ldots, B_{-1}$ of $G^{D}$ are such that $B_{-i}$ is of odd length and $B_{-i+1}, \ldots, B_{-1}$ are of even length at least 4 , then for all $y, G^{D}$ has a path with endvertices $(-1, y+1)$ and $(-1, y+2)$ that consists of all vertices of rows $y$ and $y+1$ and the vertices $\left(x_{-i}, y+2\right),\left(x_{-i}+1, y+2\right), \ldots,(-1, y+2)$.

Proof: For $1 \leq q \leq i-1$, let

$$
\begin{aligned}
Q_{-q}: & \left(x_{-q+1}-3, y\right),\left(x_{-q+1}-4, y\right), \ldots,\left(x_{-q}, y\right), \\
& \left(x_{-q}-1, y\right),\left(x_{-q}, y+1\right),\left(x_{-q}-1, y+1\right),\left(x_{-q}-2, y\right)
\end{aligned}
$$

and let

$$
\begin{aligned}
Q_{-i}: & \left(x_{-i+1}-3, y\right),\left(x_{-i+1}-2, y+1\right),\left(x_{-i+1}-3, y+1\right),\left(x_{-i+1}-4, y\right), \\
& \left(x_{-i+1}-5, y\right),\left(x_{-i+1}-4, y+1\right),\left(x_{-i+1}-5, y+1\right),\left(x_{-i+1}-6, y\right), \\
& \ldots,\left(x_{-i}+2, y\right),\left(x_{-i}+3, y+1\right),\left(x_{-i}+2, y+1\right),\left(x_{-i}+1, y\right) .
\end{aligned}
$$

Furthermore, let for $1 \leq q \leq i-1$

$$
\begin{aligned}
P_{-q}: & \left(x_{-q}, y+2\right),\left(x_{-q}+1, y+2\right), \\
& \left(x_{-q}+2, y+2\right),\left(x_{-q}+1, y+1\right),\left(x_{-q}+2, y+1\right),\left(x_{-q}+3, y+2\right), \\
& \left(x_{-q}+4, y+2\right),\left(x_{-q}+3, y+1\right),\left(x_{-q}+4, y+1\right),\left(x_{-q}+5, y+2\right), \\
& \ldots,\left(x_{-q+1}-2, y+2\right),\left(x_{-q+1}-3, y+1\right),\left(x_{-q+1}-2, y+1\right),\left(x_{-q+1}-1, y+2\right) .
\end{aligned}
$$

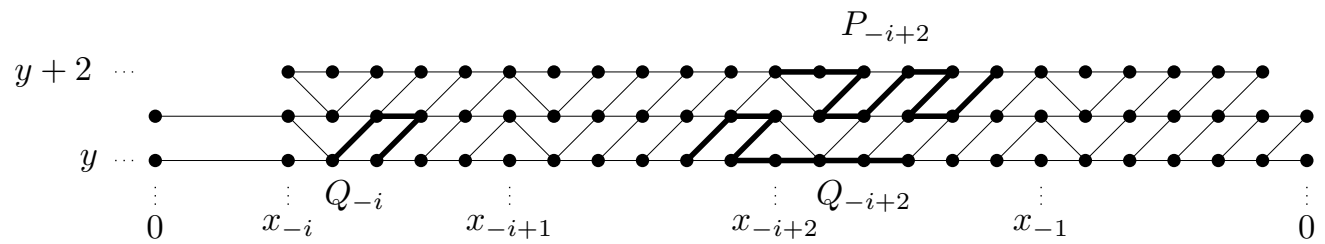

Fig. 17: $Q_{-i}, Q_{-i+2}$, and $P_{-i+2}$ for $i=4$.

Now, the sequence

$$
\begin{aligned}
& (-1, y+1),(-2, y), \\
& Q_{-1}, Q_{-2}, \ldots, Q_{-i}, \\
& \left(x_{-i}, y\right),\left(x_{-i}-1, y\right), \ldots,(-1, y), \\
& (0, y+1),(1, y+1), \ldots,\left(x_{-i}+1, y+1\right), \\
& \left(x_{-i}, y+2\right),\left(x_{-i}+1, y+2\right), \ldots,\left(x_{-i+1}-1, y+2\right), \\
& P_{-i+1}, P_{-i+2}, \ldots, P_{-1}
\end{aligned}
$$


defines a path that satisfies the conditions of the lemma. See Figures 17 and 18 for an illustration.

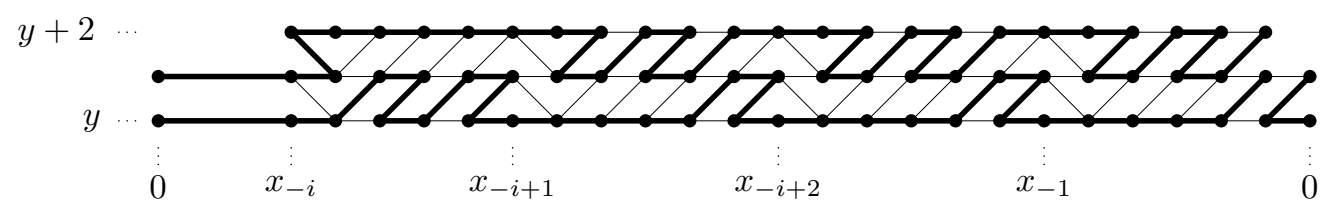

Fig. 18: A path for $i=4$.

A cycle $C$ in $G^{D}$ is called special, if $V(C)=[\min (V(C)), \max (V(C))]$.

Lemma 16 For every $D=\left\{d_{1}, d_{2}\right\} \subseteq \mathbb{N}$ with $d_{1}>d_{2}, d_{1} d_{2}$ even, and $\operatorname{gcd}\left(d_{1}, d_{2}\right)=1$, there is some $n \in \mathbb{N}$ with $n \equiv 0 \quad\left(\bmod d_{1}+d_{2}\right)$ such that $G^{D}$ has a special cycle $C$ of order $n+1$ with $V(C)=[0, n]$.

Proof: Clearly, vertex $n$ is in column 0 . Since $d_{1} d_{2}$ is even and $\operatorname{gcd}\left(d_{1}, d_{2}\right)=1$, we obtain that $d_{1}+d_{2}$ is odd and hence the number of blocks of odd length is odd, i.e. at least 1 . Let $i \in \mathbb{Z} / d_{2} \mathbb{Z}$, such that block $B_{i}$ is of odd length and the blocks $B_{i+1}, \ldots, B_{-1}$ are of even length. Clearly, by Lemma 3 , the length of the blocks $B_{i+1}, \ldots, B_{-1}$ are at least 4 . By Lemma $15, G^{D}$ has a path $Q$ with endvertices $(-1,1)$ and $(-1,2)$ that consists of all vertices of rows 0 and 1 and the vertices $\left(x_{i}, 2\right),\left(x_{i}+1,2\right), \ldots,(-1,2)$. Since the number of blocks of $B_{0}, \ldots, B_{i-1}$ of odd length is even, by Lemma $14, G^{D}$ has an $\left(x_{i}, 2\right)-\left(0, y^{\prime}\right)$ path-collection $\mathcal{R}$ for some $y^{\prime}$. Note, that if $G^{D}$ has only one block of odd length, then $\mathcal{R}=\emptyset$. In this case we define $y^{\prime}=2$. Let

$$
P=\left(Q \cup \bigcup_{R \in \mathcal{R}} R\right)+\bigcup_{y=1}^{y^{\prime}-2}\{\{(-1, y),(0, y+1)\}\}
$$

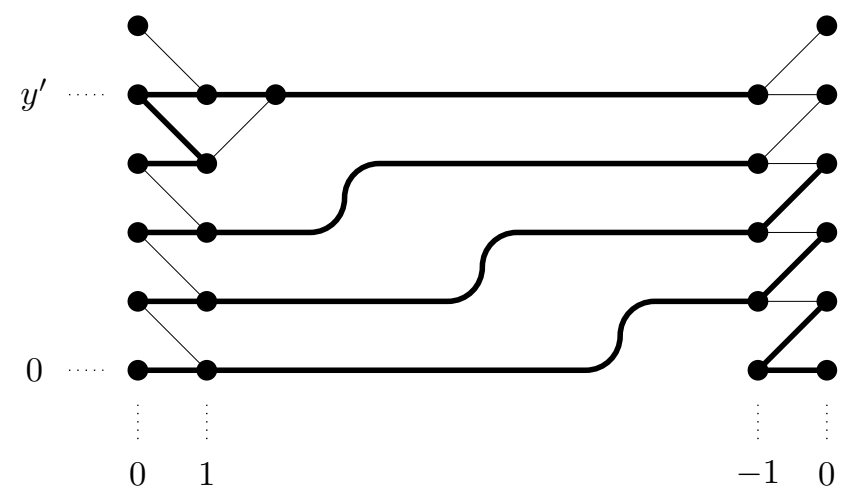

Fig. 19: The path $P$. 
By construction, $P$ is a path with endvertices $\left(-1, y^{\prime}-1\right)$ and $\left(-1, y^{\prime}\right)$ that consists of all vertices of rows $0,1, \ldots, y^{\prime}$. The vertex $\left(0, y^{\prime}\right)$ has the neighbors $\left(1, y^{\prime}-1\right)$ and $\left(1, y^{\prime}\right)$ in $P$. Since the vertex $\left(1, y^{\prime}\right)$ is an upper vertex, $\left(1, y^{\prime}\right)$ has the neighbors $\left(0, y^{\prime}\right)$ and $\left(2, y^{\prime}\right)$ in $P$ and $\left\{\left(1, y^{\prime}-1\right),\left(2, y^{\prime}\right)\right\} \in E\left(G^{D}\right)$. Now,

$$
\begin{aligned}
C= & P \\
& +\left\{\left\{\left(1, y^{\prime}-1\right),\left(2, y^{\prime}\right)\right\},\left\{\left(-1, y^{\prime}-1\right),\left(0, y^{\prime}\right)\right\},\left\{\left(-1, y^{\prime}\right),\left(0, y^{\prime}+1\right)\right\},\left\{\left(0, y^{\prime}+1\right),\left(1, y^{\prime}\right)\right\}\right\} \\
& -\left\{\left\{\left(1, y^{\prime}-1\right),\left(0, y^{\prime}\right)\right\},\left\{\left(1, y^{\prime}\right),\left(2, y^{\prime}\right)\right\}\right\}
\end{aligned}
$$

is a special cycle of $G^{D}$ of order $n+1$ with $n=\left(y^{\prime}+1\right)\left(d_{1}+d_{2}\right)$ and $V(C)=[0, n]$. See Figures 19 and 20 for an illustration.

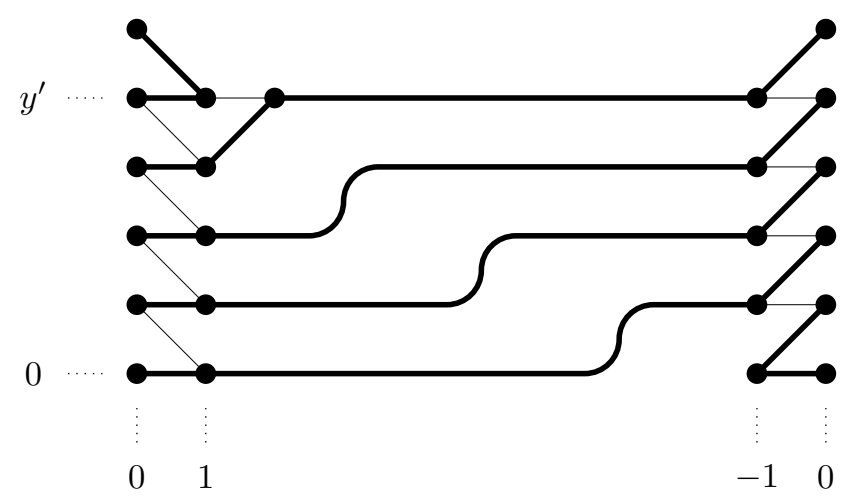

Fig. 20: The cycle $C$ in the proof of Lemma 16

Lemma 17 For every $D=\left\{d_{1}, d_{2}\right\} \subseteq \mathbb{N}$ with $d_{1}>d_{2}, d_{1} d_{2}$ odd, and $\operatorname{gcd}\left(d_{1}, d_{2}\right)=1$, there is some $n \in \mathbb{N}$ with $n \equiv 0 \quad\left(\bmod d_{1}+d_{2}\right)$ such that $G^{D}$ has a special cycle $C$ of order $n+2$ with $V(C)=[0, n+1]$.

Proof: Clearly, vertex $n$ is in column 0 . First we assume that $d_{2}=1$. In that case, $G^{D}$ has only one block and the vertex $n+1$ is in column -1 . Let $P=\emptyset$ for $d_{1}=3$, otherwise let

$$
\begin{aligned}
P: & (1,0),(2,1),(3,1),(2,0), \\
& (3,0),(4,1),(5,1),(4,0), \\
& \ldots,(-5,0),(-4,1),(-3,1),(-4,0) .
\end{aligned}
$$

The sequence

$$
C:(0,0), P,(-3,0),(-2,0),(-1,1),(-2,1),(-1,2),(0,2),(1,1),(0,1),(-1,0),(0,0)
$$

defines a special cycle of $G^{D}$ of order $2\left(d_{1}+d_{2}\right)+2$ with $V(C)=\left[0,2\left(d_{1}+d_{2}\right)+1\right]$. See Figure 21 for an illustration. 


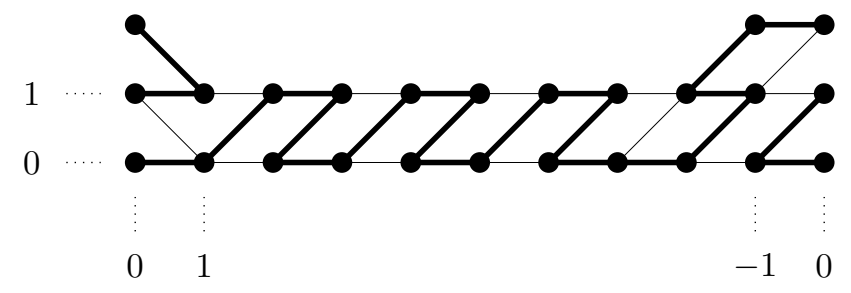

Fig. 21: The special cycle $C$ for $d_{1}=9$ and $d_{2}=1$.

Now we assume that $d_{2}>1$. Hence, by Lemma $3, G^{D}$ has more than one block. This implies that vertex $n+1$ is lower. Let $k \in \mathbb{Z} / d_{2} \mathbb{Z}$, such that vertex $n+1$ belongs to block $B_{k}$. Since $d_{1}+d_{2}$ is even, exactly those vertices are even integers that are in a column with an even index. Since vertex $n+1$ is lower and an odd integer, the number of blocks among $B_{k}, B_{k+1}, \ldots, B_{-1}$ of odd length is odd, i.e. at least one. Let $i \in \mathbb{Z} / d_{2} \mathbb{Z}$ be such that block $B_{i}$ is of odd length and the blocks $B_{i+1}, B_{i+2}, \ldots, B_{-1}$ are of even length. Clearly, by Lemma 3 , the length of the blocks $B_{i+1}, B_{i+2}, \ldots, B_{-1}$ are at least 4 . By Lemma 15. $G^{D}$ has a path $Q_{1}$ with endvertices $(-1,1)$ and $(-1,2)$ that consists of all vertices of rows 0 and 1 and the vertices $\left(x_{i}, 2\right),\left(x_{i}+1,2\right), \ldots,(-1,2)$. Since the number of blocks of $B_{k}, B_{k+1}, \ldots, B_{i-1}$ of odd length is even, by Lemma 14, $G^{D}$ has an $\left(x_{i}, 2\right)-\left(x_{k}, y^{\prime}\right)$-path-collection $\mathcal{R}_{1}$ for some $y^{\prime}$. Note, that if $i=k$, then $\mathcal{R}_{1}=\emptyset$. In this case we define $y^{\prime}=2$. By the same arguments, $G^{D}$ has a path $Q_{2}$ with endvertices $\left(-1, y^{\prime}+2\right)$ and $\left(-1, y^{\prime}+3\right)$ that consists of all vertices of rows $y^{\prime}+1$ and $y^{\prime}+2$ and the vertices $\left(x_{i}, y^{\prime}+3\right),\left(x_{i+1}, y^{\prime}+3\right), \ldots,\left(-1, y^{\prime}+3\right)$ and $G^{D}$ has an $\left(x_{i}, y^{\prime}+3\right)-\left(x_{k}, 2 y^{\prime}+1\right)$-path-collection $\mathcal{R}_{2}$.

By definition, for every $y^{\prime}+1 \leq y \leq 2 y^{\prime}$, the edges $\{(0, y),(1, y)\}$ and $\left\{\left(x_{k}-1, y\right),\left(x_{k}, y\right)\right\}$ belong to $Q_{2}$ or a path in $\mathcal{R}_{2}$. Furthermore, the path

$$
P_{0}:\left(x_{k}+1,2 y^{\prime}\right),\left(x_{k}, 2 y^{\prime}+1\right),\left(x_{k}+1,2 y^{\prime}+1\right),\left(x_{k}+2,2 y^{\prime}+1\right)
$$

is a subpath of a path in $\left\{Q_{2}\right\} \cup \mathcal{R}_{2}$. Let

$$
P_{1}:\left(0, y^{\prime}\right),\left(1, y^{\prime}\right), \ldots,\left(x_{k}-1, y^{\prime}\right)
$$

and let

$$
\begin{aligned}
P_{2}: \quad & \left(x_{k}, 2 y^{\prime}+1\right),\left(x_{k}+1,2 y^{\prime}+1\right),\left(x_{k}, 2 y^{\prime}+2\right), \\
& \left(x_{k}-1,2 y^{\prime}+1\right),\left(x_{k}-2,2 y^{\prime}+1\right), \ldots,\left(1,2 y^{\prime}+1\right) .
\end{aligned}
$$

Now,

$$
\begin{aligned}
C= & \left(Q_{1} \cup Q_{2} \cup \mathcal{R}_{1} \cup \mathcal{R}_{2}\right) \\
& -\left(E\left(P_{0}\right) \cup \bigcup_{y=y^{\prime}+1}^{2 y^{\prime}}\{\{(0, y),(1, y)\}\} \cup \bigcup_{y=y^{\prime}+1}^{2 y^{\prime}}\left\{\left\{\left(x_{k}-1, y\right),\left(x_{k}, y\right)\right\}\right\}\right) \\
& +E\left(P_{1}\right) \cup E\left(P_{2}\right) \\
& +\bigcup_{y=1}^{y^{\prime}}\{\{(-1, y),(0, y+1)\}\}
\end{aligned}
$$




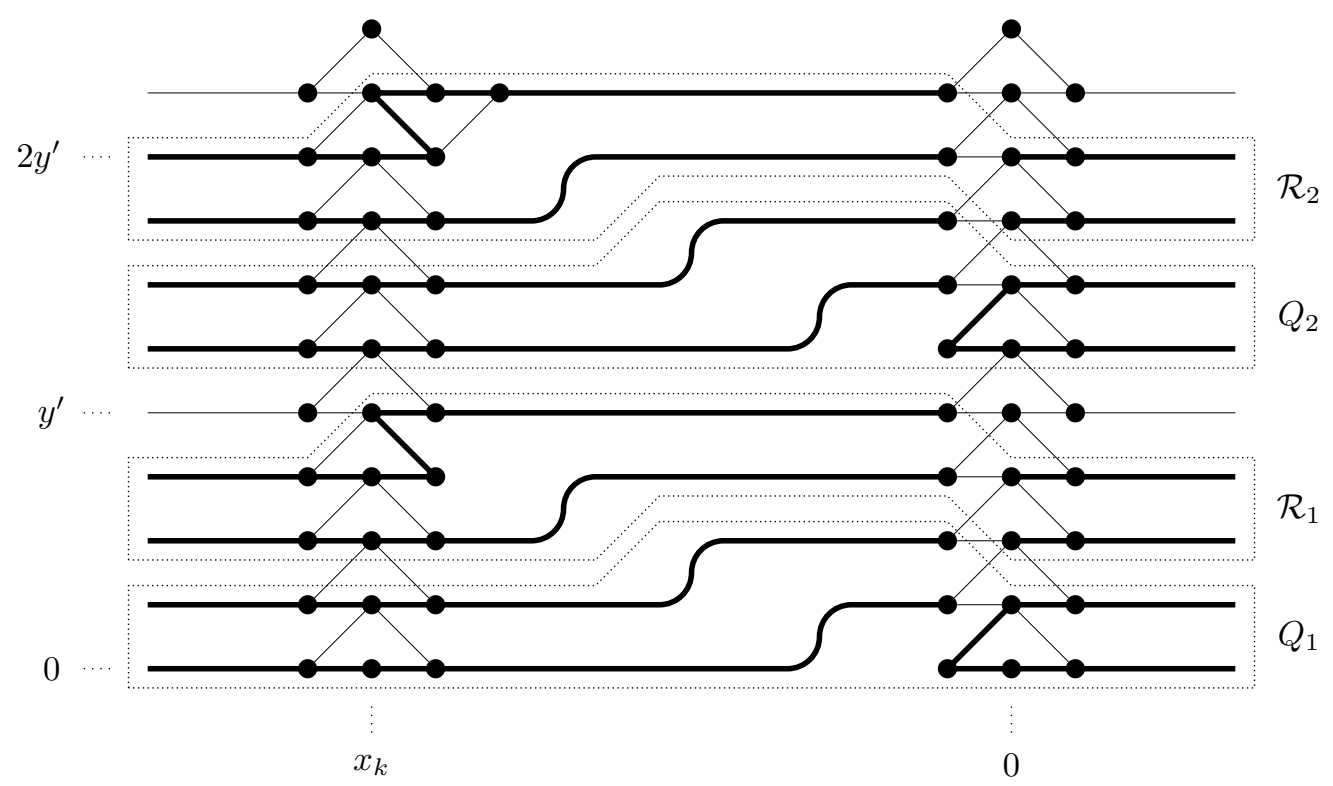

Fig. 22: $Q_{1} \cup Q_{2} \cup \mathcal{R}_{1} \cup \mathcal{R}_{2}$.

$$
\begin{aligned}
& +\bigcup_{y=y^{\prime}}^{2 y^{\prime}}\left\{\left\{\left(x_{k}-1, y\right),\left(x_{k}, y+1\right)\right\}\right\} \\
& +\bigcup_{y=y^{\prime}+2}^{2 y^{\prime}+1}\{\{(-1, y),(0, y+1)\}\} \\
& +\bigcup_{y=y^{\prime}+1}^{2 y^{\prime}+1}\{\{(0, y+1),(1, y)\}\} \\
& +\left\{\left\{\left(x_{k}+1,2 y^{\prime}\right),\left(x_{k}+2,2 y^{\prime}+1\right)\right\}\right\}
\end{aligned}
$$

defines a special cycle of $G^{D}$ of order $n+2$ with $n=\left(2 y^{\prime}+2\right)\left(d_{1}+d_{2}\right)+2$ and $V(C)=[0, n+1]$. See Figures 22 and 23 for an illustration.

Let $C$ be a special cycle of $G^{D}$ and let $n^{\prime}=\max (V(C))$. If for all $a, b \in V(C)$ with $n^{\prime}-d_{1}+1 \leq a<$ $b \leq n^{\prime},\{a, b\} \neq\left\{n^{\prime}-2 d_{2}, n^{\prime}-d_{2}\right\}$, and $|a-b| \in D$, we have $\{a, b\} \in E(C)$, then we call $C$ good.

We are now in a position to prove the main results of this section.

Proof of Theorem 9: If $D=\{1,3\}$, then the result follows by induction on $n$. $C: 0,1,2,3,0$ is a Hamiltonian cycle of $G_{4}^{D}$. Let $C_{n}$ be a Hamiltonian cycle of $G_{n}^{D}$. Since the vertex $n-1$ has degree 2 in 


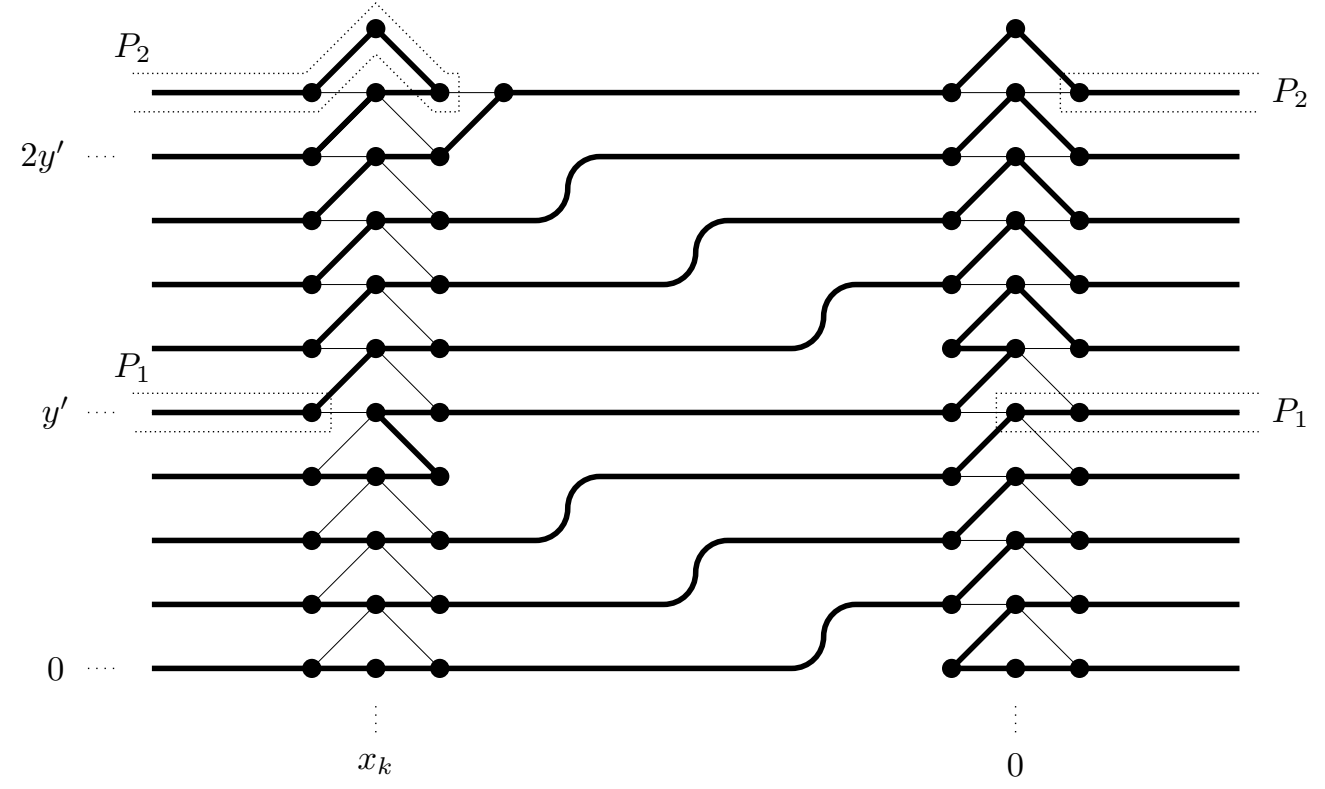

Fig. 23: The cycle $C$ in the proof of Lemma 17

$G_{n}^{D},\{n-2, n-1\} \in E\left(C_{n}\right)$. Hence,

$$
C_{n+2}=C_{n}+\{\{n-2, n+1\},\{n+1, n\},\{n, n-1\}\}-\{\{n-2, n-1\}\}
$$

is a Hamiltonian cycle of $G_{n+2}^{D}$.

Hence we can assume that $D \neq\{1,3\}$. Note that we can shift special cycles: If $C: v_{0}, \ldots, v_{l}, v_{0}$ is a special cycle in $G^{D}$, then also $C+h: v_{0}+h, \ldots, v_{l}+h, v_{0}+h$ is a special cycle in $G^{D}$. Furthermore, we can merge special cycles: If $C_{1}$ and $C_{2}$ are special cycles with $\min \left(V\left(C_{2}\right)\right)=\max \left(V\left(C_{1}\right)\right)+1$, $\{a, b\} \in E\left(C_{1}\right),\{c, d\} \in E\left(C_{2}\right)$, and $\{a, c\},\{b, d\} \in E\left(G^{D}\right)$, then

$$
\left(C_{1} \cup C_{2}\right)+\{\{a, c\},\{b, d\}\}-\{\{a, b\},\{c, d\}\}
$$

is a special cycle with vertex set $\left[\min \left(V\left(C_{1}\right)\right), \max \left(V\left(C_{2}\right)\right)\right]$. If for $i \leq a<b \leq j,\{a, b\}$ is an edge of $G^{D}$ and at least one of $a, b$ has degree 2 in $G^{D}[[i, j]]$, then the edge $\{a, b\}$ belongs to every special cycles $C$ of $G^{D}$ with $V(C)=[i, j]$.

Claim 1 If $C_{1}$ and $C_{2}$ are good special cycles of $G^{D}$ with $\min \left(V\left(C_{2}\right)\right)=\max \left(V\left(C_{1}\right)\right)+1$ and $D \neq$ $\{1,3\}$, then there is a good special cycle $C$ with $V(C)=\left[\min \left(V\left(C_{1}\right)\right), \max \left(V\left(C_{2}\right)\right)\right]$.

Proof of Claim 1: Let $n^{\prime}=\max \left(V\left(C_{1}\right)\right)$.

Case $1 d_{1} \neq 2 d_{2}+1$. 
Since $d_{1} \neq 2 d_{2}+1$ and $C_{1}$ is good, $e_{1}=\left\{n^{\prime}-d_{1}+1, n^{\prime}-d_{1}+d_{2}+1\right\} \in E\left(C_{1}\right)$. Clearly, $e_{2}=\left\{n^{\prime}+1, n^{\prime}+d_{2}+1\right\} \in E\left(C_{2}\right)$. Hence,

$$
C=\left(C_{1} \cup C_{2}\right)+\left\{\left\{n^{\prime}-d_{1}+1, n^{\prime}+1\right\},\left\{n^{\prime}-d_{1}+d_{2}+1, n^{\prime}+d_{2}+1\right\}\right\}-\left\{e_{1}, e_{2}\right\}
$$

is a good special cycle with $V(C)=\left[\min \left(V\left(C_{1}\right)\right), \max \left(V\left(C_{2}\right)\right)\right]$. This concludes the first case.

Case $2 d_{1}=2 d_{2}+1$.

Since $D \neq\{1,3\}$, we have $d_{2}>1$. Since $d_{1}=2 d_{2}+1$, and $C_{1}$ is good, $e_{1}=\left\{n^{\prime}-d_{1}+2, n^{\prime}-d_{1}+\right.$ $\left.d_{2}+2\right\} \in E\left(C_{1}\right)$. Since $d_{2}>1, e_{2}=\left\{n^{\prime}+2, n^{\prime}+d_{2}+2\right\} \in E\left(C_{2}\right)$. Hence,

$$
C=\left(C_{1} \cup C_{2}\right)+\left\{\left\{n^{\prime}-d_{1}+2, n^{\prime}+2\right\},\left\{n^{\prime}-d_{1}+d_{2}+2, n^{\prime}+d_{2}+2\right\}\right\}-\left\{e_{1}, e_{2}\right\}
$$

is a good special cycle with $V(C)=\left[\min \left(V\left(C_{1}\right)\right), \max \left(V\left(C_{2}\right)\right)\right]$. This concludes the second case and the proof of Claim 1 .

Claim $2 G^{D}$ has a good special cycle of order $2 \quad\left(\bmod d_{1}+d_{2}\right)$.

Proof of Claim 2: By Lemma 17, $G^{D}$ has a special cycle of order $2 \quad\left(\bmod d_{1}+d_{2}\right)$. Let $C_{1}$ be a special cycle of $G^{D}$ of order $2\left(\bmod d_{1}+d_{2}\right)$ and let $n^{\prime}=\max \left(V\left(C_{1}\right)\right)$. It follows from [25, 21] that $G^{D}$ has a special cycle of order $d_{1}+d_{2}$. Note that every vertex in $\left\{j, j+1, \ldots, j+d_{1}+d_{2}-1\right\}$ has degree 2 in $G^{D}\left[\left[j, j+d_{1}+d_{2}-1\right]\right]$, for $j \in \mathbb{Z}$ and hence a special cycle of order $d_{1}+d_{2}$ is good. Let $C_{2}$ be a special cycle of $G^{D}$ of order $d_{1}+d_{2}$ with $\min \left(V\left(C_{2}\right)\right)=n^{\prime}+1$. Since vertex $n^{\prime}$ has degree 2 in $G^{D}\left[V\left(C_{1}\right)\right],\left\{n^{\prime}-d_{2}, n^{\prime}\right\} \in E\left(C_{1}\right)$ and since vertex $n^{\prime}+d_{1}$ has degree 2 in $G^{D}\left[V\left(C_{2}\right)\right]$, $\left\{n^{\prime}+d_{1}-d_{2}, n^{\prime}+d_{1}\right\} \in E\left(C_{2}\right)$. Hence,

$$
\left(C_{1} \cup C_{2}\right)+\left\{\left\{n^{\prime}-d_{2}, n^{\prime}+d_{1}-d_{2}\right\},\left\{n^{\prime}, n^{\prime}+d_{1}\right\}\right\}-\left\{\left\{n^{\prime}-d_{2}, n^{\prime}\right\},\left\{n^{\prime}+d_{1}-d_{2}, n^{\prime}+d_{1}\right\}\right\}
$$

is a good special cycle of $G^{D}$. This concludes the proof of Claim 2

Let $p_{1}$ with $p_{1} \equiv 2 \quad\left(\bmod d_{1}+d_{2}\right)$, such that $G^{D}$ has a good special cycle of order $p_{1}$. By Claim 2, such a $p_{1}$ exists. As said before, $G^{D}$ has a good special cycle of order $p_{2}=d_{1}+d_{2}$. Since $\operatorname{gcd}\left(p_{1}, p_{2}\right)=2$, it follows from the extended Euclidean algorithm that every sufficiently large even integer is a positive integral linear combination of $p_{1}$ and $p_{2}$. Therefore and by Claim 11, the desired result follows by shifting and merging copies of good special cycles of order $p_{1}$ and $p_{2}$.

Proof of Theorem 10: : The proof is analogous to the proof of Theorem 9. Instead of using Lemma 17 we use Lemma 16 Proceeding as in the proof of Theorem 9 we obtain $p_{1}$ with $p_{1} \equiv 1 \quad\left(\bmod d_{1}+d_{2}\right)$ and hence $\operatorname{gcd}\left(p_{1}, p_{2}\right)=1$. This clearly allows to establish the theorem for all sufficiently large $n$ and not just for sufficiently large even $n$.

\section{Acknowledgements}

Christian Löwenstein and Dieter Rautenbach acknowledge partial support from the DFG project "Cycle Spectra of Graphs" RA873/5-1. Roman Soták acknowledges support by the Slovak Science and Technology Assistance Agency under the contract APVV-0023-10. 


\section{References}

[1] T. Araki and Y. Shibata. Pancyclicity of recursive circulant graphs. Inf. Process. Lett., 81:187-190, 2002.

[2] J.-C. Bermond, F. Comellas, and D. F. Hsu. Distributed loop computer networks: A survey. J. of Parallel and Distributed Computing, 24:2-10, 1985.

[3] D.K. Biss. Hamiltonian decomposition of recursive circulant graphs. Discrete Math., 214:89-99, 2000.

[4] F. Boesch and R. Tindell. Circulants and their connectivities. J. Graph Theory, 8:487-499, 1984.

[5] Z.R. Bogdanowicz. Pancyclicity of connected circulant graphs. J. Graph Theory, 22:167-174, 1996.

[6] G.J. Chang, L. Huang, and X. Zhu. Circular chromatic numbers and fractional chromatic numbers of distance graphs. Europ. J. Combinatorics, 19:423-431, 1998.

[7] M. Chih Lin, D. Rautenbach, F.J. Soulignac, and J.L. Szwarcfiter. Powers of Cycles, Powers of Paths, and Distance Graphs. Discrete Appl. Math., 159:621-627, 2011.

[8] W. Deuber and X. Zhu. The chromatic number of distance graphs. Discrete Math., 165/166:195-204, 1997.

[9] B. Effantin and H. Kheddouci. The b-chromatic number of some power graphs. Discrete Math. Theor. Comput. Sci., 6:45-54, 2003.

[10] R.B. Eggleton, P. Erdős, and D.K. Skilton. Coloring the real line. J. Combin. Theory Ser. B, 39:86$100,1985$.

[11] R.B. Eggleton, P. Erdôs, and D.K. Skilton. Colouring prime distance graphs. Graphs Combin., 6:1732, 1990 .

[12] S.A. Evdokimov and I.N. Ponomarenko. Circulant graphs: Recognizing and isomorphism testing in polynomial time. St. Petersbg. Math. J., 15:813-835, 2004.

[13] D. Gómez, J. Gutierrez, and Á. Ibeas. Connectedness of finite distance graphs. Networks, 60:204-209, 2012.

[14] H. Harborth and S. Krause. Distance Ramsey numbers. Util. Math., 70:197-200, 2006.

[15] C. Heuberger. On Hamiltonian Toeplitz graphs. Discrete Math., 245:107-125, 2002.

[16] F.K. Hwang. A complementary survey on double-loop networks. Theoret. Comput. Sci., 263:211229, 2001.

[17] F.K. Hwang. A survey on multi-loop networks. Theoret. Comput. Sci., 299:107-121, 2003.

[18] A. Kemnitz and M. Marangio. Colorings and list colorings of integer distance graphs. Congr. Numerantium, 151:75-84, 2001. 
[19] A. Kemnitz and M. Marangio. Chromatic numbers of integer distance graphs. Discrete Math., 233:239-246, 2001.

[20] K. Kutnar and D. Marušič. Hamilton cycles and paths in vertex-transitive graphs - current directions. Discrete Math., 309:5491-5500, 2009.

[21] C. Löwenstein, D. Rautenbach, and F. Regen. On Hamiltonian Paths in Distance Graphs. Appl. Math. Lett., 24:1075-1079, 2011.

[22] M. Muzychuk. A solution of the isomorphism problem for circulant graphs. Proc. Lond. Math. Soc., III. Ser., 88:1-41, 2004.

[23] L.D. Penso, D. Rautenbach, and J.L. Szwarcfiter. Cycles, Paths, Connectivity and Diameter in Distance Graphs. LNCS, 5911:320-328, 2010.

[24] L.D. Penso, D. Rautenbach, and J.L. Szwarcfiter. Connectivity and Diameter in Distance Graphs. Networks, 57:310-315, 2011.

[25] L.D. Penso, D. Rautenbach, and J.L. Szwarcfiter. Long Cycles and Paths in Distance Graphs. Discrete Math., 310:3417-3420, 2010.

[26] C.S. Raghavendra and J.A. Sylvester. A survey of multi-connected loop topologies for local computer networks. Comput. Network ISDN Syst., 11:29-42, 1986.

[27] R. van Dal, G. Tijssen, Zs. Tuza, J.A. van der Veen, C. Zamfirescu, and T. Zamfirescu. Hamiltonian properties of Toeplitz graphs. Discrete Math., 159:69-81, 1996.

[28] M. Voigt. Colouring of distance graphs. Ars Combin., 52:3-12, 1999. 